\title{
The 2005 IALLT Survey of the Profession
}

In the following pages you'll find the results of IALLT's Survey of the Profession, conducted in May of 2005. The survey netted 116 respondents from a variety of institutions, from large research universities to liberal arts colleges to community colleges to a few public K-12 schools and special institutions, such as the Foreign Service Institute.

The survey was put together by David Pankratz (Loyola University Chicago) and Andrew Ross (Brown University), based on the format and general subject outlines of the 2001 IALLT Survey by Monika Dressler (University of Michigan). The programming of the survey instrument itself was ably, quickly and cheerfully done by Mark van Hoek (University of Victoria).

What this is

\section{What this isn't}

The 2005 IALLT Survey of the Profession is a snapshot of language resource centers, language labs, language and media centers, etc. We heard from 116 institutions primarily in the United States and Canada, though there are a few responses from overseas institutions. As a snapshot, it passes along to you only what's in the picture frame, not what's outside it. We have attempted to provide an overview of the field as reported by our respondents - your mileage may vary. The responses are the answers of language center directors to a wide range of questions, aggregated by institutional type so that comparisons can be made both within and across categories. We will no doubt have failed to ask some questions that have become critical in the intervening year (do you podcast language materials on a regular basis?). Rest assured that we will ask these questions in subsequent surveys, and that we will communicate the results to you in a timely fashion.

The 2005 IALLT Survey of the Profession is not polemic, massaged, spun or interpreted. It is data with a minimum of interference by the editorial staff involved in its production. You'll note that there's not a lot of text - no global conclusions drawn, no calls for movement on administrative, technical or political fronts. This is deliberate, as we wanted to get the information gathered in the survey out to you as quickly as possible. Parsing the data to find correlations, anomalies and trends is time-consuming and beyond the scope of this particular publication, which hopes to be factual rather than interpretative. 
A word about the organization of the survey results is in order: the responses to the survey have been divided into subsets which correspond to the self-identified Carnegie classification type of the responding institution ${ }^{1}$. These are:

Doctoral Extensive

Doctoral Intensive

Master's ${ }^{2}$

Baccalaureate - Liberal Arts

Baccalaureate - General

Associate's (Community College)

Special Institution

We have also included a K-12 category not listed in Carnegie's system of classification. For detailed information about each of the classification categories listed above, please see http://www.educause.edu/CarnegieClassifications/1051. It was decided to organize the responses according to these rubrics so that members at a given institution can compare their situations to others in their general institutional category as well as to the respondent population as a whole. In the tables that follow, each category has its own column, with overall figures and percentages in bold at the lefthand side of the page.

Here's a basic example of the tabular results that you'll see in the survey:

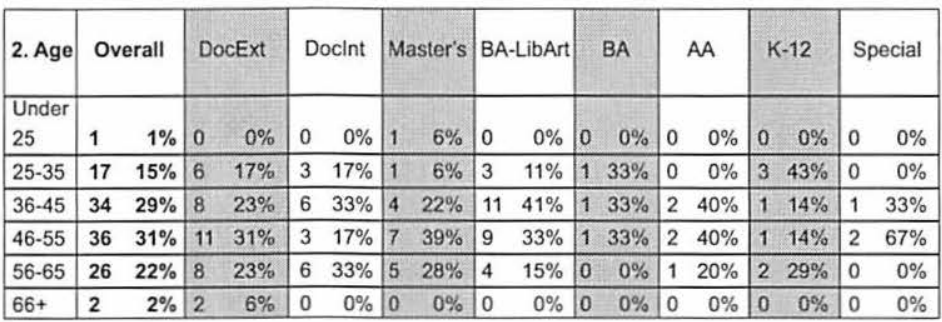

The numbers and percentages in bold indicate the overall results returned prior to categorization by institutional type. Reading down the "Baccalaureate - Liberal Arts" column, you'll be able to note that $41 \%$ of respondents were between 36 and 45 years of age, compared to $29 \%$ overall. Most questions follow this general layout - a few are Likert scale ( 1 to 5, strongly disagree to strongly agree, none to frequently).

Note that this survey uses a subset of the 2000 Carnegie classifications rather than the new classifications (2005). The next IALLT Survey will use the 2005 classifications to organize response data.

2The Master's category was collapsed from two subcategories (Master's I and Master's II), given the small $\mathrm{n}$. 
Here's an example using the Likert scale:

31. Indleate the lovel of use of the following materials within your center. If your center does not have the Item N/A. For results by category, see Appendix B.

\begin{tabular}{|c|c|c|c|c|c|c|c|c|c|c|}
\hline \multirow[b]{2}{*}{ Audio Cassettes } & \multicolumn{2}{|c|}{ None } & \multicolumn{2}{|c|}{ Infrequent } & \multicolumn{2}{|c|}{ Moderato } & \multicolumn{2}{|c|}{ Frequtent } & \multicolumn{2}{|c|}{ N/A } \\
\hline & 10 & $9 \%$ & 41 & $38 \%$ & 27 & $23 \%$ & & $16 \%$ & & $\mathbf{9 \%}$ \\
\hline Audio CD & 3 & $3 \%$ & 32 & $28 \%$ & 32 & $28 \%$ & 41 & $35 \%$ & 3 & $3 \%$ \\
\hline Digitized Audio for Streaming & 4 & $3 \%$ & 8 & $7 \%$ & 15 & $13 \%$ & 68 & $59 \%$ & 13 & $11 \%$ \\
\hline Videotapes & 4 & $3 \%$ & 13 & $11 \%$ & 36 & $31 \%$ & 54 & $47 \%$ & 5 & $4 \%$ \\
\hline OVD & 1 & $1 \%$ & 11 & $9 \%$ & 40 & $34 \%$ & 53 & $46 \%$ & 5 & $4 \%$ \\
\hline Digitized Video for Streamino & 9 & $8 \%$ & 27 & $23 \%$ & 29 & $25 \%$ & 28 & $24 \%$ & 18 & $16 \%$ \\
\hline CD-ROM & 2 & $2 \%$ & 27 & $23 \%$ & 39 & $34 \%$ & 38 & $33 \%$ & & $4 \%$ \\
\hline $\begin{array}{l}\text { Computer applications available } \\
\text { in your center only }\end{array}$ & 3 & $3 \%$ & 11 & $9 \%$ & 38 & $33 \%$ & 56 & $48 \%$ & 3 & $3 \%$ \\
\hline $\begin{array}{l}\text { Computer applications avdilable } \\
\text { campus-wide }\end{array}$ & 7 & $6 \%$ & 12 & $10 \%$ & 25 & $22 \%$ & 47 & $41 \%$ & 17 & $15 \%$ \\
\hline Slides & 37 & $32 \%$ & 37 & $32 \%$ & 7 & $6 \%$ & 0 & $0 \%$ & 28 & $24 \%$ \\
\hline Maps & 36 & $31 \%$ & 35 & $30 \%$ & 11 & $9 \%$ & 2 & $2 \%$ & 26 & $22 \%$ \\
\hline Books, periodicals & 20 & $17 \%$ & 40 & $34 \%$ & 27 & $23 \%$ & 9 & $8 \%$ & 14 & $12 \%$ \\
\hline Satellite Programming & & $11 \%$ & 24 & $21 \%$ & 26 & $22 \%$ & 28 & $24 \%$ & 17 & $15 \%$ \\
\hline $\begin{array}{l}\text { Self-Study/ Independent Study } \\
\text { Materials (analog or digital) }\end{array}$ & 4 & $3 \%$ & 32 & $28 \%$ & 32 & $28 \%$ & 34 & $29 \%$ & 7 & $6 \%$ \\
\hline
\end{tabular}

The above are overall results from all institutional categories (note that they're in bold). Results for this question by Carnegie classification will be found in an appendix to the survey, so that you can compare your reponses to those of other institutions that are at least nominally similar. Note that "None" in this question does not mean that your center has none of the item in question; rather, it has the item but makes no use of it. A response of "N/A" here and elsewhere indicates that your center does not have the item or provide the service referred to in the question.

Additional Comments:

The IALLT Survey provided limited space for text responses to Question 58 ("What are the most important issues your center will face during the next several years? Please use keywords only.") at the end of the instrument. We have included verbatim responses to Question 58 - note that they are reprinted exactly as received, capitalizations, usage and misspellings intact. Below is a brief resumé of the responses, organized by keyword and theme (special thanks to David Pankratz for putting this together): 
Support

Need for more institutional support (staff, funding, etc.) 41 responses ( $48 \%$ of 85 total respondents)

\section{Structural change}

Change from "physical center" to "virtual center" 22 responses ( $26 \%$ of respondents)

Conversion from analog to digital technologies 15 responses ( $18 \%$ of respondents)

Renovation, adaptation, repurposing of center 15 responses (18\% of respondents)

\section{Operational maintenance}

Equipment and materials acquisition and maintenance 20 responses ( $24 \%$ of respondents)

\section{Pedagogy}

Curriculum, teaching methodology, pedagogy 15 responses $(18 \%$ of respondents)

Note: Total of percentages exceeds 100 because some respondents mentioned more than one topic.

Other topics mentioned several times are:

Center's mission

Relationship with Information Technologies department

Role of publishers' materials

We hope the Survey will be useful to the profession as a whole, and to you personally.

Please don't hesitate to contact the survey authors with questions and comments: Andrew Ross (Andrew Ross@brown.edu), David Pankratz (dpankra@luc.edu). 


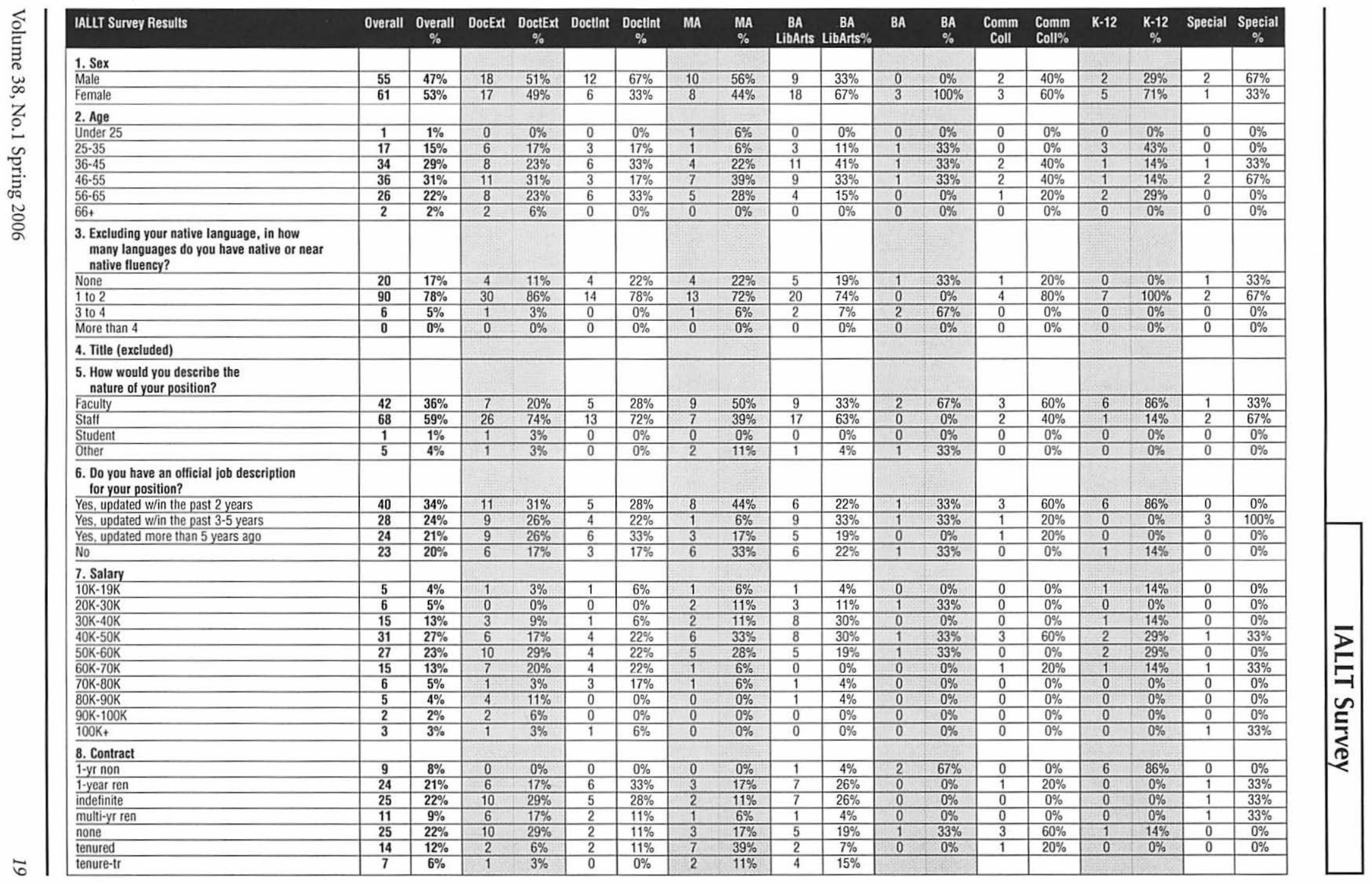


BABBS

MANequiv

PhD/equiv

\begin{tabular}{|l|l|l|l|l|l|l|l|l|l|l|l|l|l|l|}
\hline 3 & $3 \%$ & 0 & $0 \%$ & 0 & $0 \%$ & 0 & $0 \%$ & 1 & $4 \%$ & & $14 \%$ & 0 & $0 \%$ \\
\hline
\end{tabular}

9a. Degree Type

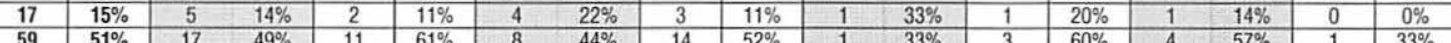

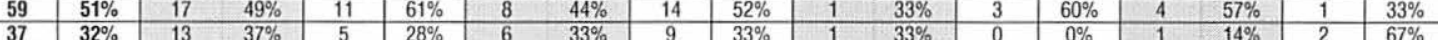

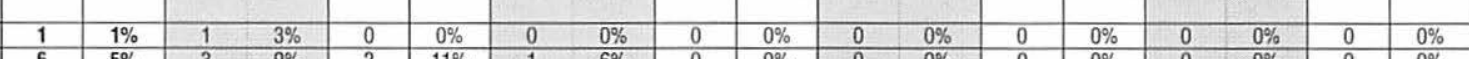
\begin{tabular}{|l|c|c|c|c|c|c|c|c|c|c|c|c|c|c|c|c|c|c|c|c|}
\hline Inglish & 6 & $5 \%$ & 3 & $9 \%$ & 2 & $11 \%$ & 1 & $6 \%$ & 0 & $0 \%$ & 0 & $0 \%$ & 0 & $0 \%$ & 0 & $0 \%$ & 0 & $0 \%$ \\
\hline $\mathrm{LL}$ & 57 & $49 \%$ & 21 & $60 \%$ & 7 & $39 \%$ & 7 & $39 \%$ & 13 & $48 \%$ & 2 & $67 \%$ & 2 & $40 \%$ & 4 & $57 \%$ & 1 & $33 \%$ \\
\hline
\end{tabular}

$\frac{\text { Hum }}{\text { CompSci }}$

Com

LocSci

\section{K-12 Certification}

No

Yes

11. Years in current position

$\frac{0-1}{2+05}$

$\frac{2105}{61010}$

$\frac{611 \text { to } 15}{161020}$

$\frac{1610}{21 \text { t0 }}$

$25+$

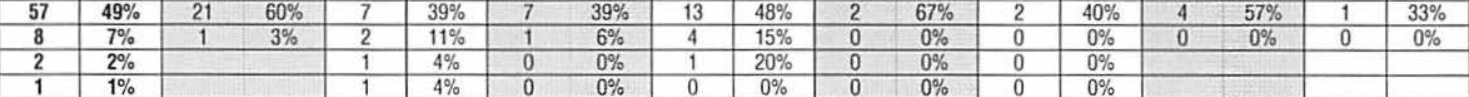

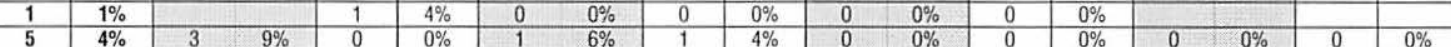

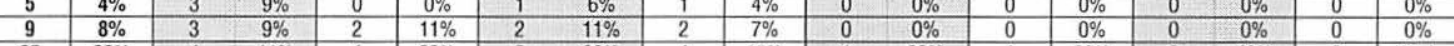

\section{What general activities are regular tasks for}

your current position within the language lab/

Attend regional / national / international

professional conferences

Present papers at professional conferences

Engage in academic publication

books, articles, reviews, etc.

Regularly teach foreign language courses Regularly teach hiterature / cultural studies courses Research and implement technological innovations Research and implem Plan tacility's budget

The lacilys desin Collaborate with programs outside of the institution Begularly participate on institutional committees Write or collaborate on internal and

Write or collaborate on in
external grant proposals

external grant proposals

Publicize projects and events

Build / manage audio and video materials collections Create / interpret copyright policy for your facility

\begin{tabular}{|c|c|c|c|c|c|c|c|c|c|c|c|c|c|c|c|c|c|}
\hline 25 & $22 \%$ & 4 & $11 \%$ & 4 & $22 \%$ & 6 & $33 \%$ & 4 & $15 \%$ & 1 & $33 \%$ & 1 & $20 \%$ & 3 & $43 \%$ & 2 & $67 \%$ \\
\hline & & & & & & & & & & & & & & & & & \\
\hline 85 & $73 \%$ & 27 & $77 \%$ & 11 & $61 \%$ & 13 & $72 \%$ & 23 & $85 \%$ & 2 & $67 \%$ & 3 & $60 \%$ & 3 & $43 \%$ & 3 & $100 \%$ \\
\hline 30 & $26 \%$ & 8 & $23 \%$ & 6 & $33 \%$ & 5 & $28 \%$ & 4 & $15 \%$ & 1 & $33 \%$ & 2 & $40 \%$ & 4 & $57 \%$ & 0 & $0 \%$ \\
\hline 1 & $1 \%$ & 0 & 0 & 1 & $6 \%$ & 0 & $0 \%$ & 0 & $0 \%$ & 0 & $0 \%$ & 0 & $0 \%$ & 0 & $0 \%$ & 0 & $0 \%$ \\
\hline & & & & & & & & & & & & & & & & & \\
\hline 9 & $8 \%$ & 3 & $9 \%$ & 1 & $6 \%$ & 3 & $17 \%$ & 0 & $0 \%$ & 1 & $33 \%$ & 1 & $20 \%$ & 0 & $0 \%$ & 0 & $0 \%$ \\
\hline 42 & $36 \%$ & 10 & $29 \%$ & 7 & $39 \%$ & 5 & $28 \%$ & 12 & $44 \%$ & 1 & $33 \%$ & 2 & $40 \%$ & 4 & $57 \%$ & 1 & $33 \%$ \\
\hline 27 & $23 \%$ & 9 & $26 \%$ & 4 & $22 \%$ & 5 & $28 \%$ & 5 & $19 \%$ & 0 & $0 \%$ & 1 & $20 \%$ & 1 & $14 \%$ & 2 & $67 \%$ \\
\hline 13 & $11 \%$ & 3 & $9 \%$ & 1 & $6 \%$ & 3 & $17 \%$ & 5 & $19 \%$ & 1 & $33 \%$ & 0 & $0 \%$ & 0 & $0 \%$ & 0 & $0 \%$ \\
\hline 14 & $12 \%$ & 4 & $11 \%$ & 3 & $17 \%$ & 2 & $11 \%$ & 2 & $7 \%$ & 0 & $0 \%$ & 1 & $20 \%$ & 2 & $29 \%$ & 0 & $0 \%$ \\
\hline 5 & $4 \%$ & 3 & $9 \%$ & 0 & $0 \%$ & 0 & $0 \%$ & 2 & $7 \%$ & 0 & $0 \%$ & 0 & $0 \%$ & 0 & $0 \%$ & 0 & $0 \%$ \\
\hline 6 & $5 \%$ & 3 & $9 \%$ & 2 & $11 \%$ & 0 & $0 \%$ & 1 & $4 \%$ & 0 & $0 \%$ & 0 & $0 \%$ & 0 & $0 \%$ & 0 & $0 \%$ \\
\hline 97 & $84 \%$ & 29 & $83 \%$ & 17 & $94 \%$ & 14 & $78 \%$ & 26 & $96 \%$ & 2 & $67 \%$ & 3 & $60 \%$ & 3 & $43 \%$ & 3 & $100 \%$ \\
\hline 52 & $45 \%$ & 23 & $66 \%$ & 8 & $44 \%$ & 5 & $28 \%$ & 10 & $37 \%$ & 2 & $67 \%$ & 1 & $20 \%$ & 1 & $14 \%$ & 2 & $67 \%$ \\
\hline 38 & $33 \%$ & 12 & $34 \%$ & 6 & $33 \%$ & 7 & $39 \%$ & 8 & $30 \%$ & 2 & $67 \%$ & 1 & $20 \%$ & 1 & $14 \%$ & 1 & $33 \%$ \\
\hline 50 & $43 \%$ & 5 & $14 \%$ & 7 & $39 \%$ & 11 & $61 \%$ & 15 & $56 \%$ & 2 & $67 \%$ & 2 & $40 \%$ & 7 & $100 \%$ & 1 & $33 \%$ \\
\hline 12 & $10 \%$ & 1 & $3 \%$ & 0 & $0 \%$ & 4 & $22 \%$ & 6 & $22 \%$ & 1 & $33 \%$ & 0 & $0 \%$ & 0 & $0 \%$ & 0 & $0 \%$ \\
\hline 17 & $15 \%$ & 9 & $26 \%$ & 3 & $17 \%$ & 1 & $6 \%$ & 3 & $11 \%$ & 0 & $0 \%$ & 0 & $0 \%$ & 0 & $0 \%$ & 1 & $33 \%$ \\
\hline 92 & $79 \%$ & 27 & $77 \%$ & 15 & $83 \%$ & 14 & $78 \%$ & 25 & $93 \%$ & 3 & $100 \%$ & 4 & $80 \%$ & 1 & $14 \%$ & 3 & $100 \%$ \\
\hline 80 & $69 \%$ & 29 & $83 \%$ & 15 & $83 \%$ & 7 & $39 \%$ & 18 & $67 \%$ & 2 & $67 \%$ & 4 & $80 \%$ & 3 & $43 \%$ & 2 & $67 \%$ \\
\hline 92 & $79 \%$ & 29 & $83 \%$ & 15 & $83 \%$ & 14 & $78 \%$ & 22 & $81 \%$ & 3 & $100 \%$ & 5 & $100 \%$ & 2 & $29 \%$ & 2 & $67 \%$ \\
\hline 84 & $72 \%$ & 31 & $89 \%$ & 11 & $61 \%$ & 12 & $67 \%$ & 20 & $74 \%$ & 1 & $33 \%$ & 5 & $100 \%$ & 1 & $14 \%$ & 3 & $100 \%$ \\
\hline 51 & $44 \%$ & 19 & $54 \%$ & 10 & $56 \%$ & 6 & $33 \%$ & 9 & $33 \%$ & 1 & $33 \%$ & 3 & $60 \%$ & 1 & $14 \%$ & 2 & $67 \%$ \\
\hline 73 & $63 \%$ & 23 & $66 \%$ & 14 & $78 \%$ & 12 & $67 \%$ & 13 & $48 \%$ & 2 & $67 \%$ & 3 & $60 \%$ & 3 & $43 \%$ & 3 & $100 \%$ \\
\hline 66 & $57 \%$ & 24 & $69 \%$ & 9 & $50 \%$ & 11 & $61 \%$ & 13 & $48 \%$ & 1 & $33 \%$ & 5 & $100 \%$ & 2 & $29 \%$ & 1 & $33 \%$ \\
\hline 70 & $60 \%$ & 25 & $71 \%$ & 17 & $94 \%$ & 7 & $39 \%$ & 13 & $48 \%$ & 0 & $0 \%$ & 5 & $100 \%$ & 0 & $0 \%$ & 3 & $100 \%$ \\
\hline 22 & $19 \%$ & 10 & $29 \%$ & 1 & $6 \%$ & 4 & $22 \%$ & 0 & $0 \%$ & 1 & $33 \%$ & 1 & $20 \%$ & 2 & $29 \%$ & 3 & $100 \%$ \\
\hline 101 & $87 \%$ & 31 & $89 \%$ & 18 & $100 \%$ & 17 & $94 \%$ & 23 & $85 \%$ & 3 & $100 \%$ & 5 & $100 \%$ & 2 & $29 \%$ & 2 & $67 \%$ \\
\hline 68 & $59 \%$ & 29 & $83 \%$ & 11 & $61 \%$ & 7 & $39 \%$ & 15 & $56 \%$ & 1 & $33 \%$ & 2 & $40 \%$ & 1 & $14 \%$ & 2 & $67 \%$ \\
\hline
\end{tabular}




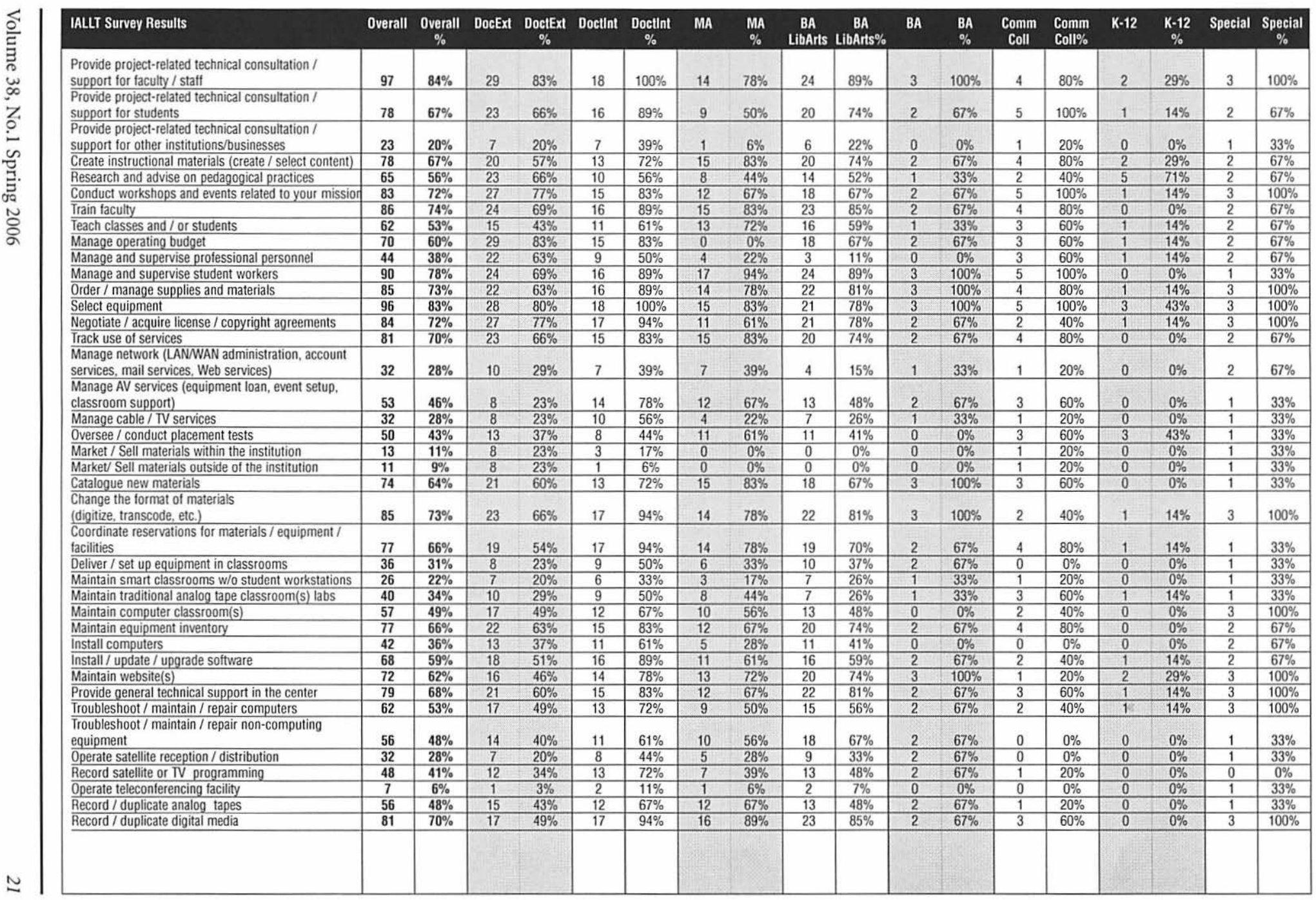


13. Whith skill areas are most important in pertosming your work successfflly?

Check only the five most importan

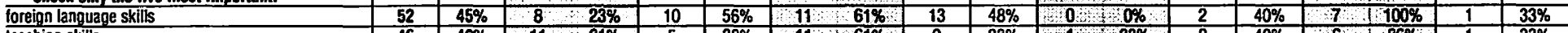

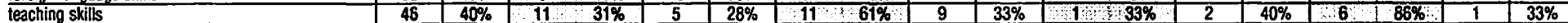
\begin{tabular}{|l|c|c|c|c|c|c|c|c|c|c|c|c|c|c|c|c|c|c|c|c|c|c|c|c|}
\hline writing skills & 22 & $19 \%$ & 9 & $26 \%$ & 3 & $17 \%$ & 3 & $17 \%$ & 5 & $19 \%$ & 0 & $0 \%$ & 2 & $40 \%$ & 0 & $0 \%$ & 0 & $0 \%$ \\
\hline
\end{tabular}

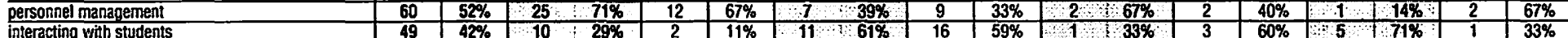

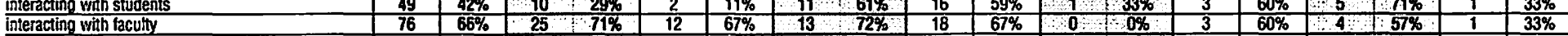

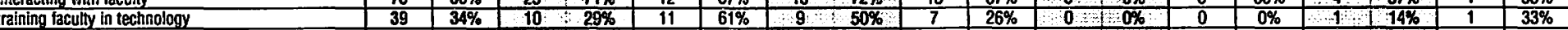

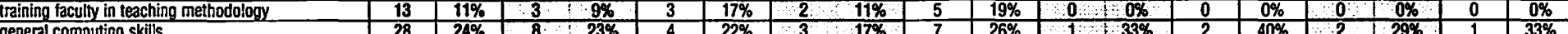

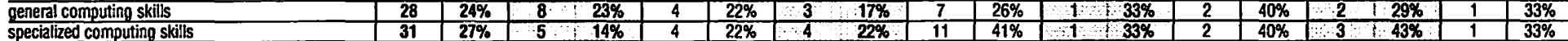

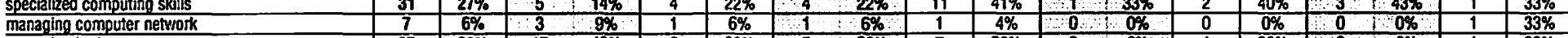

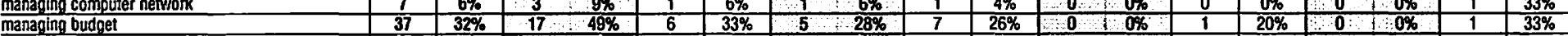

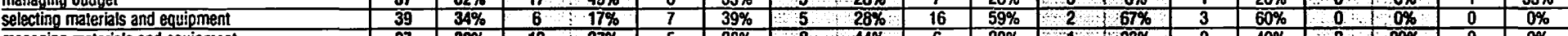
\begin{tabular}{|l|l|l|l|l|l|l|lll|l|l|l|l|l|l|l|l|l|}
\hline managing materials and equipment & 37 & $32 \%$ & 13 & $37 \%$ & 5 & $28 \%$ & 8 & $44 \%$ & 6 & $22 \%$ & 1 & $40 \%$ & 2 & $29 \%$ & 0 & $0 \%$ \\
\hline
\end{tabular}

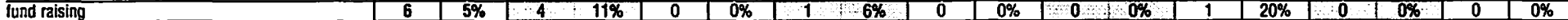
strategic planning 14. Check each type of applieation listed below that
you use on a personal computar as part of your you use on a persostion's dutias.

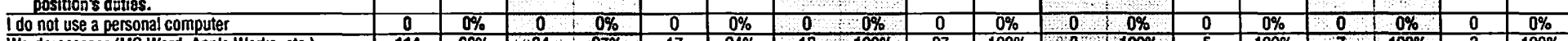

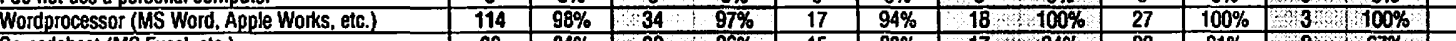

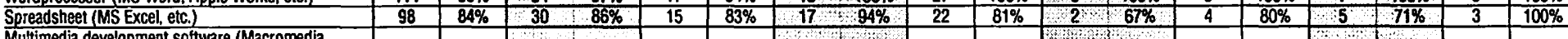
Multimedia development solware (Macromedia Director, Asymetrix Toolbook, etc.) Dre Development maine (Macrom Dreamweaver, Contribute, Adobe FrontPage, etc.) (MS Access. FileMaker Pro, etc.) Desktop Publishing

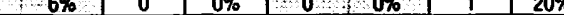
(Adobe PageMaker, Ouark Express, etc.)
Chat / Instant Messaging (AlM, NatMeeting, etc.) Audio editing software (Bias PEAK, Adobe Audition, Audacity, etc.

(Adobe Premiere, Apple Final Cut Pro, etc.) \begin{tabular}{|l|l|l|l|l|l|l|l|l|l|l|l|l|l|l|l|l|l|}
34 & $29 \%$ & 11 & $31 \%$ & 10 & $56 \%$ & 4 & $22 \%$ & 5 & $19 \%$ & 1 & $33 \%$ & 1 & $20 \%$ & 1 & $14 \%$ & 1 & $33 \%$ \\
\hline
\end{tabular} \begin{tabular}{|c|c|c|c|c|c|c|c|c|c|c|c|c|c|c|c|c|}
\hline 85 & $73 \%$ & 25 & $71 \%$ & 14 & $78 \%$ & 13 & $72 \%$ & 22 & $81 \%$ & 2 & $67 \%$ & 4 & $80 \%$ & 3 & $43 \%$ & 2 \\
\hline
\end{tabular} \begin{tabular}{|l|l|l|l|l|l|lll|l|l|l|l|l|l|l|l|l|l}
58 & $50 \%$ & 19 & $54 \%$ & 12 & $67 \%$ & 9 & $50 \%$ & 11 & $41 \%$ & 2 & $67 \%$ & 2 & $40 \%$ & 2 & $29 \%$ & 1 & $33 \%$ \\
\hline
\end{tabular}

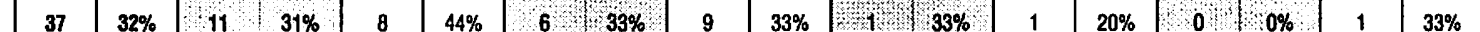
\begin{tabular}{|l|l|l|l|l|l|l|l|l|l|l|l|l|l|l|l|l|l|l|l}
37 & $32 \%$ & 11 & $31 \%$ & 8 & $44 \%$ & 6 & $33 \%$ & 9 & $33 \%$ & $0 \%$ & 1 & $33 \%$ \\
\hline 32 & $28 \%$ & 11 & $31 \%$ & 6 & $33 \%$ & 6 & $33 \%$ & 6 & $22 \%$ & 0 & $0 \%$ & 0 & $0 \%$ & 2 & $29 \%$ & 1 & $33 \%$ \\
\hline
\end{tabular}

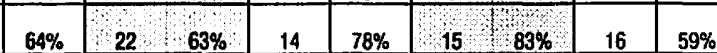

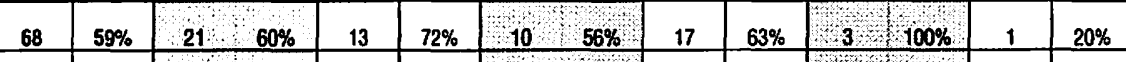
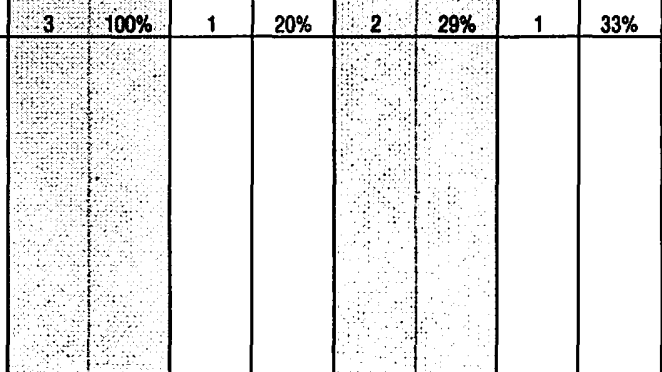


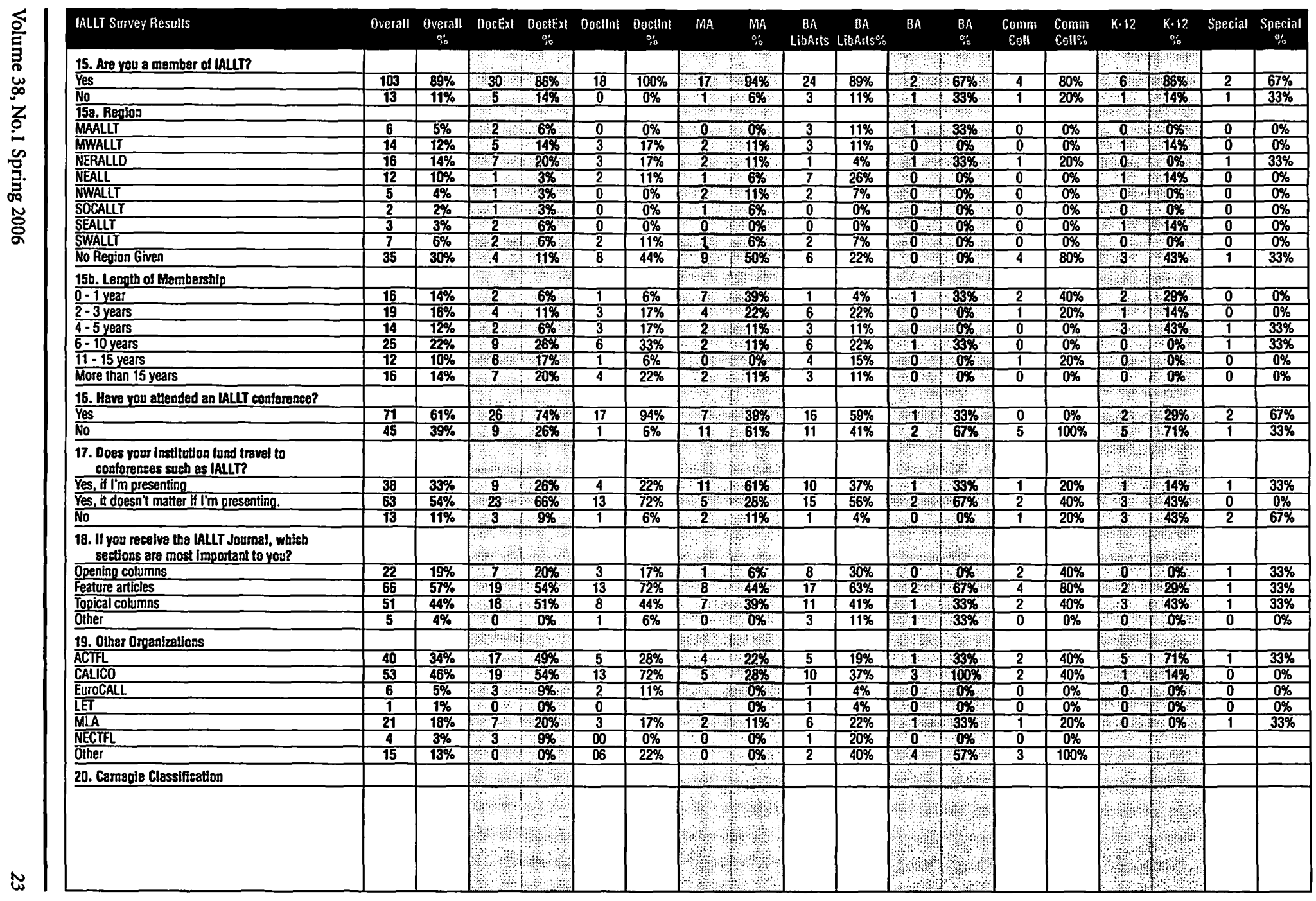




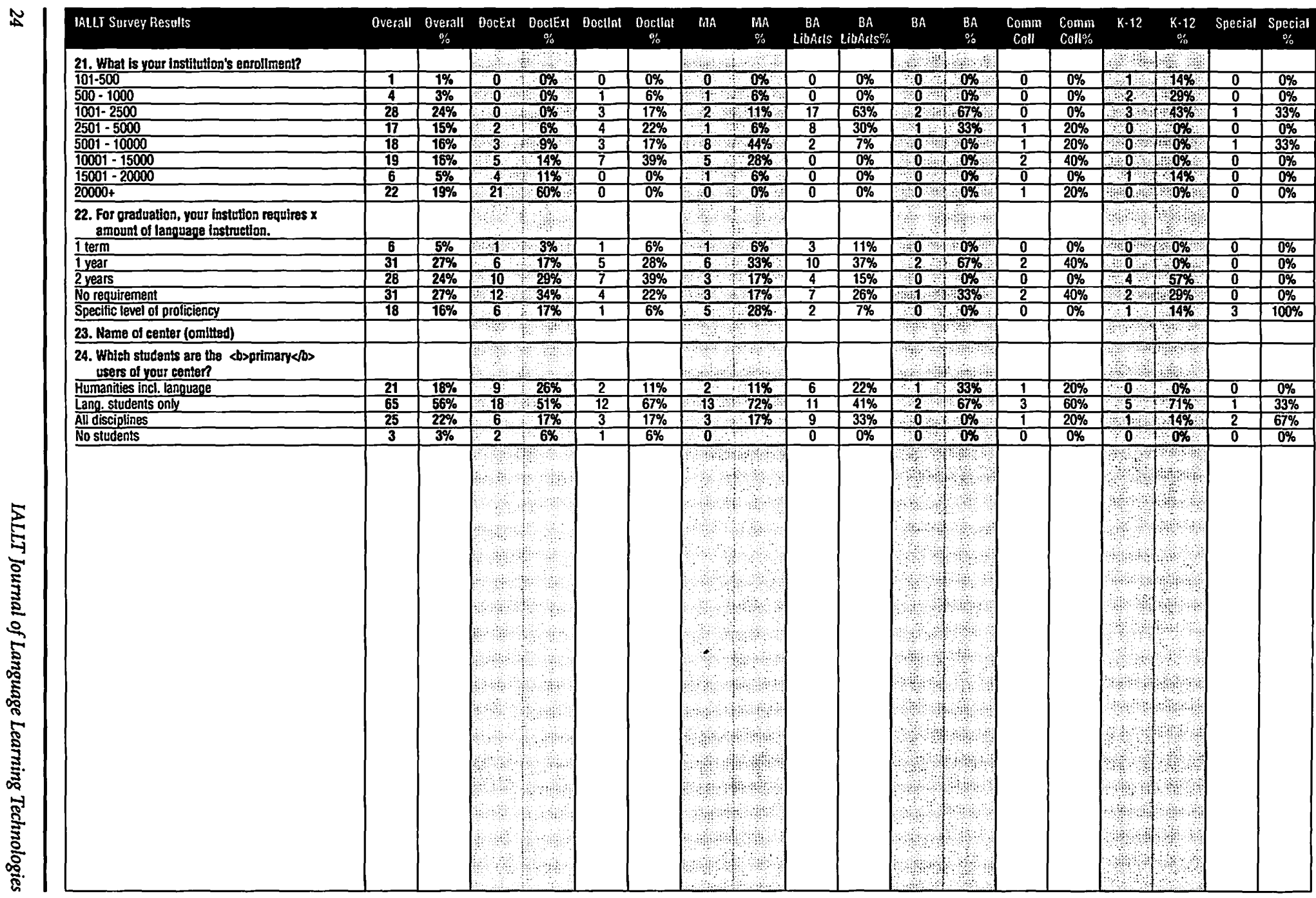




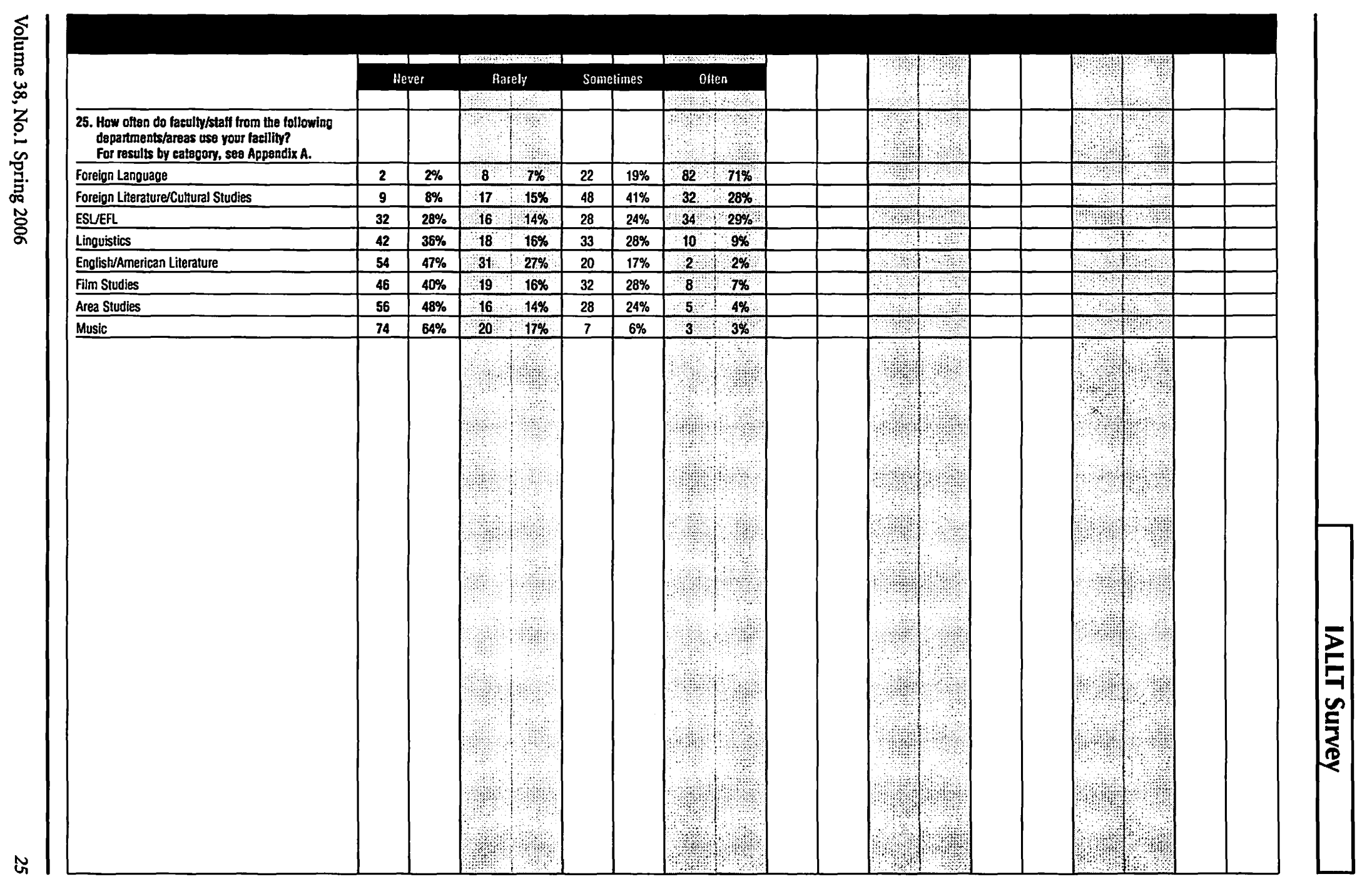




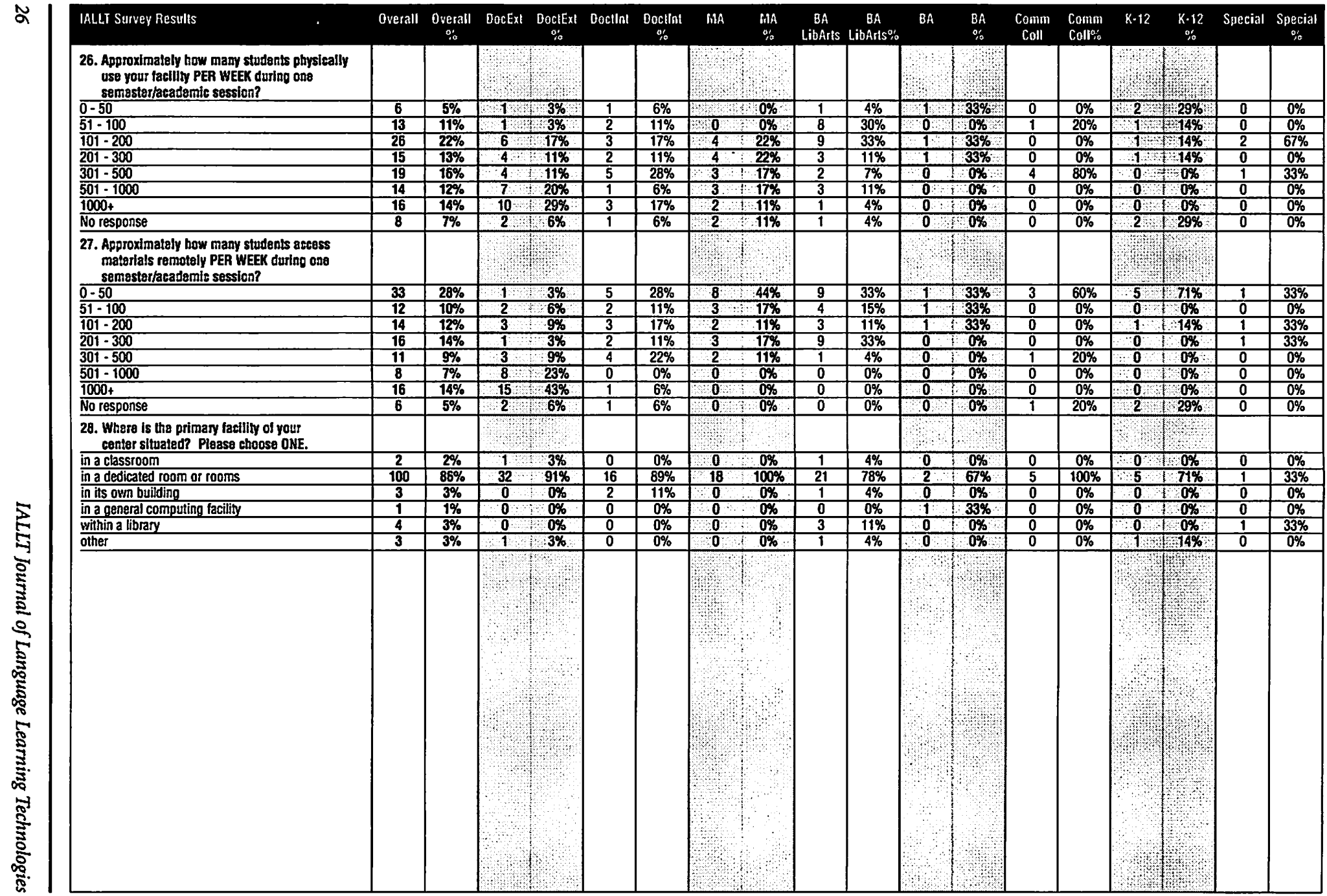




\begin{tabular}{|c|c|c|c|c|c|c|c|c|c|c|c|c|c|c|c|c|c|c|}
\hline IALIT Survey Results & Overall & $\begin{array}{c}\text { Overall } \\
\%\end{array}$ & DocExt & $\begin{array}{c}\text { DoctExt } \\
\%\end{array}$ & Doctint & $\begin{array}{c}\text { Doctint } \\
\%\end{array}$ & MA & $\begin{array}{l}\text { MA } \\
\%\end{array}$ & $\begin{array}{c}\text { BA } \\
\text { LibArts } \\
\end{array}$ & $\begin{array}{c}\text { BA } \\
\text { LibArts\% }\end{array}$ & BA & $\begin{array}{l}B A \\
\%\end{array}$ & $\begin{array}{c}\text { Comm } \\
\text { Coll }\end{array}$ & $\begin{array}{l}\text { Comm } \\
\text { Coll\% }\end{array}$ & $K-12$ & $\begin{array}{c}K-12 \\
\%\end{array}$ & Special & $\begin{array}{c}\text { Special } \\
\%\end{array}$ \\
\hline \multicolumn{19}{|l|}{$\begin{array}{l}\text { 29. Check off all areas of your center under its } \\
\text { direct supervision as dedicated space or rooms. } \\
\text { Check all that apply. }\end{array}$} \\
\hline classroom(s) & 34 & $29 \%$ & 12 & $34 \%$ & 8 & $44 \%$ & 3 & $17 \%$ & 4 & $15 \%$ & 2 & $67 \%$ & 3 & $60 \%$ & 1 & $14 \%$ & 1 & $33 \%$ \\
\hline smart classroom(s) & 37 & $32 \%$ & 14 & $40 \%$ & 7 & $39 \%$ & 3 & $17 \%$ & 10 & $37 \%$ & 1 & $33 \%$ & 1 & $20 \%$ & 0 & $0 \%$ & 1 & $33 \%$ \\
\hline computer classroom(s) & 86 & $74 \%$ & 29 & $83 \%$ & 14 & $78 \%$ & 12 & $67 \%$ & 21 & $78 \%$ & 2 & $67 \%$ & 3 & $60 \%$ & 2 & $29 \%$ & 3 & $100 \%$ \\
\hline analog audio lab classroom & 35 & $30 \%$ & 8 & $23 \%$ & 8 & $44 \%$ & 6 & $33 \%$ & 6 & $22 \%$ & 1 & $33 \%$ & 4 & $80 \%$ & 1 & $14 \%$ & 1 & $33 \%$ \\
\hline digital audio lab classroom & 50 & $43 \%$ & 15 & $43 \%$ & 5 & $28 \%$ & 9 & $50 \%$ & 11 & $41 \%$ & 3 & $100 \%$ & 2 & $40 \%$ & 4 & $57 \%$ & 1 & $33 \%$ \\
\hline video/film viewing carrels & 69 & $59 \%$ & 25 & $71 \%$ & 10 & $56 \%$ & 12 & $67 \%$ & 14 & $52 \%$ & 2 & $67 \%$ & 3 & $60 \%$ & 1 & $14 \%$ & 2 & $67 \%$ \\
\hline audio recording studio & 39 & $34 \%$ & 20 & $57 \%$ & 10 & $56 \%$ & 2 & $11 \%$ & 4 & $15 \%$ & 0 & $0 \%$ & 2 & $40 \%$ & 0 & $0 \%$ & 1 & $33 \%$ \\
\hline Staff production area for materials development & 54 & $47 \%$ & 24 & $69 \%$ & 9 & $50 \%$ & 6 & $33 \%$ & 9 & $33 \%$ & 2 & $67 \%$ & 2 & $40 \%$ & 1 & $14 \%$ & 1 & $33 \%$ \\
\hline Text Library & 28 & $24 \%$ & 11 & $31 \%$ & 5 & $28 \%$ & 3 & $17 \%$ & 6 & $22 \%$ & 0 & $0 \%$ & 2 & $40 \%$ & 0 & $0 \%$ & 1 & $33 \%$ \\
\hline Group viewing area for satellite reception & 34 & $29 \%$ & 14 & $40 \%$ & 7 & $39 \%$ & 3 & $17 \%$ & 8 & $30 \%$ & 0 & $0 \%$ & 1 & $20 \%$ & 0 & $0 \%$ & 1 & $33 \%$ \\
\hline Video Library & 76 & $66 \%$ & 24 & $69 \%$ & 13 & $72 \%$ & 14 & $78 \%$ & 16 & $59 \%$ & 3 & $100 \%$ & 3 & $60 \%$ & 0 & $0 \%$ & 3 & $100 \%$ \\
\hline Video viewing areas accommodating 3-4+ students & 55 & $47 \%$ & 21 & $60 \%$ & 7 & $39 \%$ & 9 & $50 \%$ & 13 & $48 \%$ & 2 & $67 \%$ & 2 & $40 \%$ & 0 & $0 \%$ & 1 & $33 \%$ \\
\hline Audio Library & 68 & $59 \%$ & 24 & $69 \%$ & 9 & $50 \%$ & 13 & $72 \%$ & 14 & $52 \%$ & 3 & $100 \%$ & 3 & $60 \%$ & 0 & $0 \%$ & 2 & $67 \%$ \\
\hline Software Library & 49 & $42 \%$ & 18 & $51 \%$ & 7 & $39 \%$ & 7 & $39 \%$ & 12 & $44 \%$ & 2 & $67 \%$ & 2 & $40 \%$ & 0 & $0 \%$ & 1 & $33 \%$ \\
\hline Video studio & 17 & $15 \%$ & 7 & $20 \%$ & 3 & $17 \%$ & 3 & $17 \%$ & 2 & $7 \%$ & 1 & $33 \%$ & 1 & $20 \%$ & 0 & $0 \%$ & 0 & $0 \%$ \\
\hline Repair Bench/Shop & 21 & $18 \%$ & 11 & $31 \%$ & 3 & $17 \%$ & 2 & $11 \%$ & 3 & $11 \%$ & 0 & $0 \%$ & 1 & $20 \%$ & 0 & $0 \%$ & 1 & $33 \%$ \\
\hline Closed Circuit TV & 13 & $11 \%$ & 9 & $26 \%$ & 1 & $6 \%$ & 1 & $6 \%$ & 2 & $7 \%$ & 0 & $0 \%$ & 0 & $0 \%$ & 0 & $0 \%$ & 0 & $0 \%$ \\
\hline Server Room & 44 & $38 \%$ & 18 & $51 \%$ & 8 & $44 \%$ & 7 & $39 \%$ & 6 & $22 \%$ & 0 & $0 \%$ & 2 & $40 \%$ & 1 & $14 \%$ & 2 & $67 \%$ \\
\hline Teleconferencing Studio & 6 & $5 \%$ & 2 & $6 \%$ & 1 & $6 \%$ & 1 & $6 \%$ & 0 & $0 \%$ & 0 & $0 \%$ & 1 & $20 \%$ & 1 & $14 \%$ & 0 & $0 \%$ \\
\hline Student project work area for materials development & 36 & $31 \%$ & 12 & $34 \%$ & 6 & $33 \%$ & 2 & $11 \%$ & 12 & $44 \%$ & 1 & $33 \%$ & 2 & $40 \%$ & 1 & $14 \%$ & 0 & $0 \%$ \\
\hline \multicolumn{18}{|l|}{ Faculty and/or TA project work area for } & $0 \%$ \\
\hline Meeting Room & 30 & $26 \%$ & 18 & $51 \%$ & 4 & $22 \%$ & $\frac{\pi}{3}$ & $17 \%$ & 2 & $7 \%$ & 0 & $0 \%$ & 1 & $20 \%$ & 0 & $0 \%$ & 2 & $67 \%$ \\
\hline Administrative Office & 77 & $66 \%$ & 28 & $80 \%$ & 17 & $94 \%$ & 9 & $50 \%$ & 16 & $59 \%$ & 2 & $67 \%$ & 2 & $40 \%$ & 0 & $0 \%$ & 3 & $100 \%$ \\
\hline Storage Space & 73 & $63 \%$ & 29 & $83 \%$ & 12 & $67 \%$ & 12 & $67 \%$ & 13 & $48 \%$ & 1 & $33 \%$ & 3 & $60 \%$ & 2 & $29 \%$ & 1 & $33 \%$ \\
\hline Lounge & 25 & $22 \%$ & 11 & $31 \%$ & 6 & $33 \%$ & 1 & $6 \%$ & 6 & $22 \%$ & 1 & $33 \%$ & 0 & $0 \%$ & 0 & $0 \%$ & 0 & $0 \%$ \\
\hline \multicolumn{19}{|l|}{ Other (textbox) } \\
\hline \multicolumn{19}{|l|}{$\begin{array}{l}\text { 30. Which of the following services are offered } \\
\text { within your facility under its direct supervision? } \\
\text { Check all that apply. }\end{array}$} \\
\hline Audio tape use on-site & 75 & $65 \%$ & 23 & $66 \%$ & 15 & $83 \%$ & 11 & $61 \%$ & 16 & $59 \%$ & 1 & $33 \%$ & 3 & $60 \%$ & 3 & $43 \%$ & 3 & $100 \%$ \\
\hline Audio tape checkout for home use & 32 & $28 \%$ & 13 & $37 \%$ & 5 & $28 \%$ & 3 & $17 \%$ & 6 & $22 \%$ & 0 & $0 \%$ & 2 & $40 \%$ & 1 & $14 \%$ & 2 & $67 \%$ \\
\hline Monitored drill/practice in lab sessions & 52 & $45 \%$ & 14 & $40 \%$ & 10 & $56 \%$ & 9 & $50 \%$ & 10 & $37 \%$ & 0 & $0 \%$ & 2 & $40 \%$ & 5 & $71 \%$ & 2 & $67 \%$ \\
\hline Audio Equipment Check-Out & 60 & $52 \%$ & 22 & $63 \%$ & 13 & $72 \%$ & 8 & $44 \%$ & 13 & $48 \%$ & 2 & $67 \%$ & 0 & $0 \%$ & 0 & $0 \%$ & 2 & $67 \%$ \\
\hline Audio Equipment Delivery & 14 & $12 \%$ & 1 & $3 \%$ & 5 & $28 \%$ & 3 & $17 \%$ & 4 & $15 \%$ & 1 & $33 \%$ & 0 & $0 \%$ & 0 & $0 \%$ & 0 & $0 \%$ \\
\hline Audio Equipment Staff Assistance & 47 & $41 \%$ & 19 & $54 \%$ & 10 & $56 \%$ & 5 & $28 \%$ & 9 & $33 \%$ & 2 & $67 \%$ & 0 & $0 \%$ & 1 & $14 \%$ & 1 & $33 \%$ \\
\hline Audio cassette duplication & 79 & $68 \%$ & 30 & $86 \%$ & 14 & $78 \%$ & 11 & $61 \%$ & 15 & $56 \%$ & 2 & $67 \%$ & 2 & $40 \%$ & 2 & $29 \%$ & 3 & $100 \%$ \\
\hline Audio format conversion or transfer & 69 & $59 \%$ & 23 & $66 \%$ & 14 & $78 \%$ & 9 & $50 \%$ & 16 & $59 \%$ & 2 & $67 \%$ & 0 & $0 \%$ & 3 & $43 \%$ & 2 & $67 \%$ \\
\hline Digitize audio materials for on-site use & 85 & $73 \%$ & 27 & $77 \%$ & 14 & $78 \%$ & 14 & $78 \%$ & 21 & $78 \%$ & 2 & $67 \%$ & 2 & $40 \%$ & 3 & $43 \%$ & 2 & $67 \%$ \\
\hline \multicolumn{19}{|l|}{ Digitize audio materials for remote use } \\
\hline (via web or server) & 76 & $66 \%$ & 31 & $89 \%$ & 13 & $72 \%$ & 9 & $50 \%$ & 17 & $63 \%$ & 2 & $67 \%$ & 2 & $40 \%$ & 0 & $0 \%$ & 2 & $67 \%$ \\
\hline Provide audio reserves & 51 & $44 \%$ & 19 & $54 \%$ & 8 & $44 \%$ & 6 & $33 \%$ & 12 & $44 \%$ & 3 & $100 \%$ & 2 & $40 \%$ & 0 & $0 \%$ & 1 & $33 \%$ \\
\hline Audio production (recording original material) & 65 & $56 \%$ & 25 & $71 \%$ & 14 & $78 \%$ & 7 & $39 \%$ & 10 & $37 \%$ & 2 & $67 \%$ & 4 & $80 \%$ & 1 & $14 \%$ & 2 & $67 \%$ \\
\hline \multicolumn{19}{|l|}{ Other (textbox) } \\
\hline VHS tape/DVD use on-site & 102 & $88 \%$ & 32 & $91 \%$ & 17 & $94 \%$ & 17 & $94 \%$ & 23 & $85 \%$ & 3 & $100 \%$ & 4 & $80 \%$ & 3 & $43 \%$ & 3 & $100 \%$ \\
\hline VHS tape/DVD checkout for home use & 35 & $30 \%$ & 14 & $40 \%$ & 6 & $33 \%$ & 4 & $22 \%$ & 7 & $26 \%$ & 0 & $0 \%$ & 2 & $40 \%$ & 0 & $0 \%$ & 2 & $67 \%$ \\
\hline Monitored drill/practice in lab sessions & 36 & $31 \%$ & 5 & $14 \%$ & 7 & $39 \%$ & 7 & $39 \%$ & 9 & $33 \%$ & 0 & $0 \%$ & 3 & $60 \%$ & 3 & $43 \%$ & 2 & $67 \%$ \\
\hline Video equipment check-0ut & 47 & $41 \%$ & 18 & $51 \%$ & 9 & $50 \%$ & 10 & $56 \%$ & 7 & $26 \%$ & 2 & $67 \%$ & 0 & $0 \%$ & 0 & $0 \%$ & 1 & $33 \%$ \\
\hline
\end{tabular}


Video equipment delivery

\begin{tabular}{|l|l|l|l|l|l|l|l|l|l|l|l|l|}
15 & $13 \%$ & 0 & $0 \%$ & 5 & $28 \%$ & 4 & $22 \%$ & 4 & $15 \%$ & 2 & $67 \%$ & 0 \\
\hline
\end{tabular}

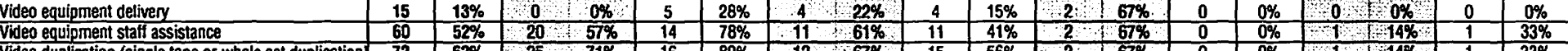

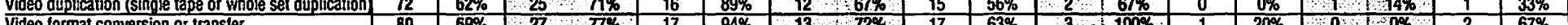

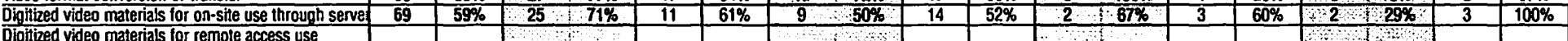
Diginzed video materials lor remole access use via vieb or server)

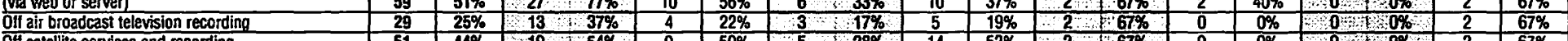

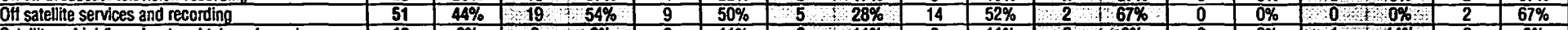

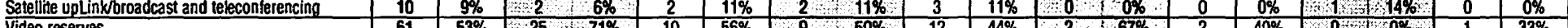

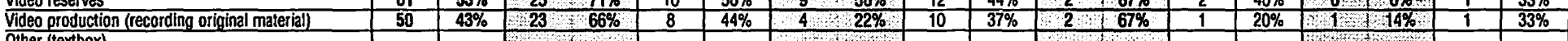
Ohar (exibox) Monitored dril Practice in lab sessions Computer use on-site

Computer equipment check-out Computer equipment delivery Compuer equmpent stall assistance Offer local applications (CD-ROMS, applications found on center computers only)

Instructional design and consultat

nsrecionaldesign and consulaton

Computer appication programiesson production

Other (textbox)



Teacher Training. Teacher Evaluation

Grant Writing

Peer/Group Tutoring

Placement Exams

Ora! Proficiency Exams

Administration of standard exams

(SAT II, AP, etc - not necessarily language-related)

\begin{tabular}{|l|l|l|l|l|l|l|l|l|l|l|l|l|l|l|l|}
\hline 48 & $41 \%$ & 10 & $29 \%$ & 7 & $39 \%$ & 10 & $56 \%$ & 12 & $44 \%$ & 0 & $0 \%$ & 4 & $80 \%$ & 4 & $33 \%$ \\
\hline
\end{tabular}

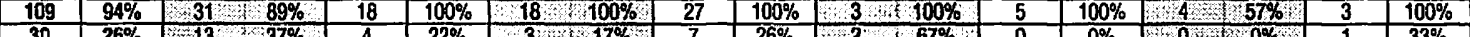
\begin{tabular}{|l|l|l|l|l|l|lll|l|l|l|l|l|l|l|l|l|}
\hline 30 & $26 \%$ & 13 & $37 \%$ & 4 & $22 \%$ & 3 & $17 \%$ & 7 & $26 \%$ & 2 & $67 \%$ & 0 & $0 \%$ & 0 & $0 \%$ & 1 & $33 \%$ \\
\hline 14 & $12 \%$ & 0 & $0 \%$ & 3 & $17 \%$ & 4 & $22 \%$ & 4 & $15 \%$ & 2 & $16 \%$ & 0 & $0 \%$ & 0 & $0 \%$ & 1 & $33 \%$ \\
\hline
\end{tabular} \begin{tabular}{|l|l|l|l|l|l|l|l|l|l|l|l|l|l|l|l|l|}
67 & $58 \%$ & 21 & $60 \%$ & 16 & $89 \%$ & 11 & $61 \%$ & 13 & $48 \%$ & 3 & $100 \%$ & 0 & $0 \%$ & 1 & 2 & $67 \%$ \\
\hline
\end{tabular} \begin{tabular}{|l|l|l|l|l|l|l|l|l|l|l|l|l|l|l|l|}
\hline 74 & $64 \%$ & 28 & $80 \%$ & 14 & $78 \%$ & 10 & $56 \%$ & 14 & $52 \%$ & 3 & $100 \%$ & 2 & $40 \%$ & 1 & 2 \\
\hline
\end{tabular}

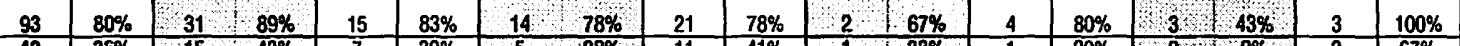

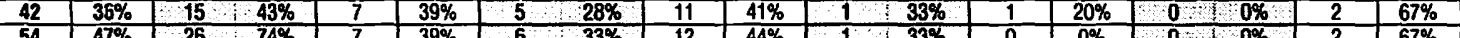

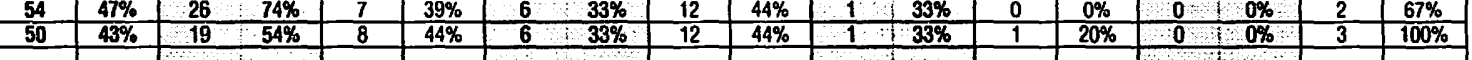

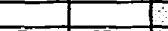

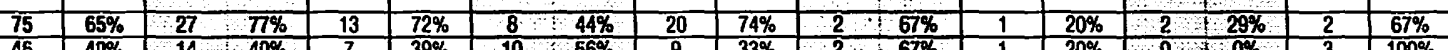

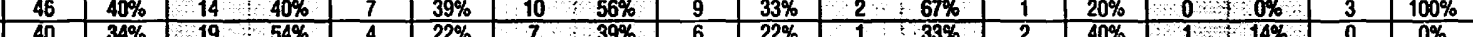

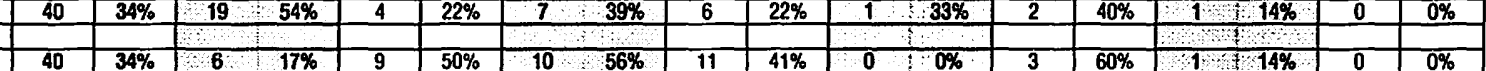

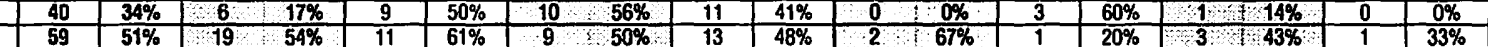

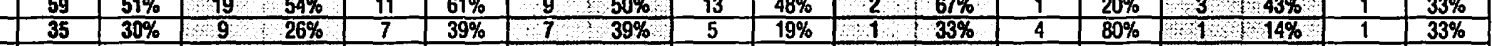
24
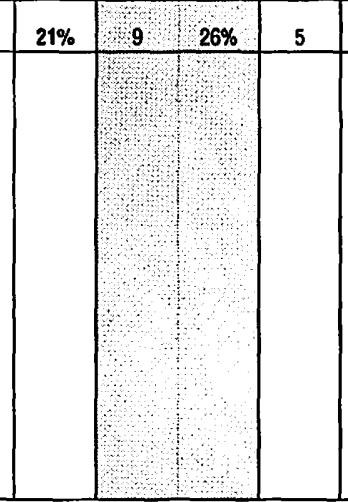

$28 \%$

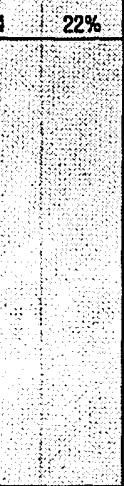

\begin{tabular}{l|l}
4 & $15 \%$ \\
\hline
\end{tabular}
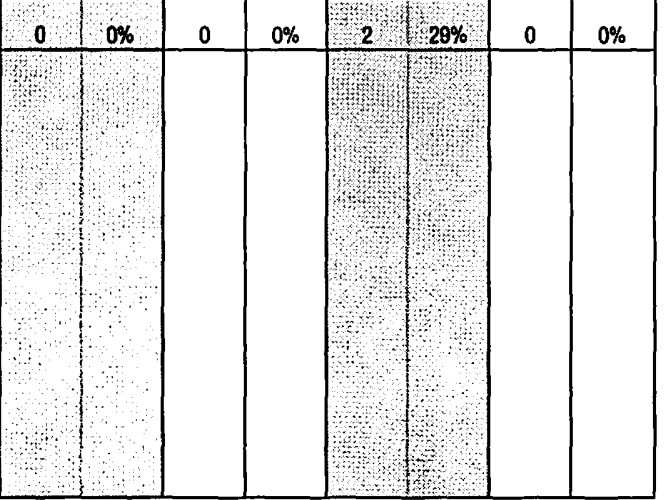


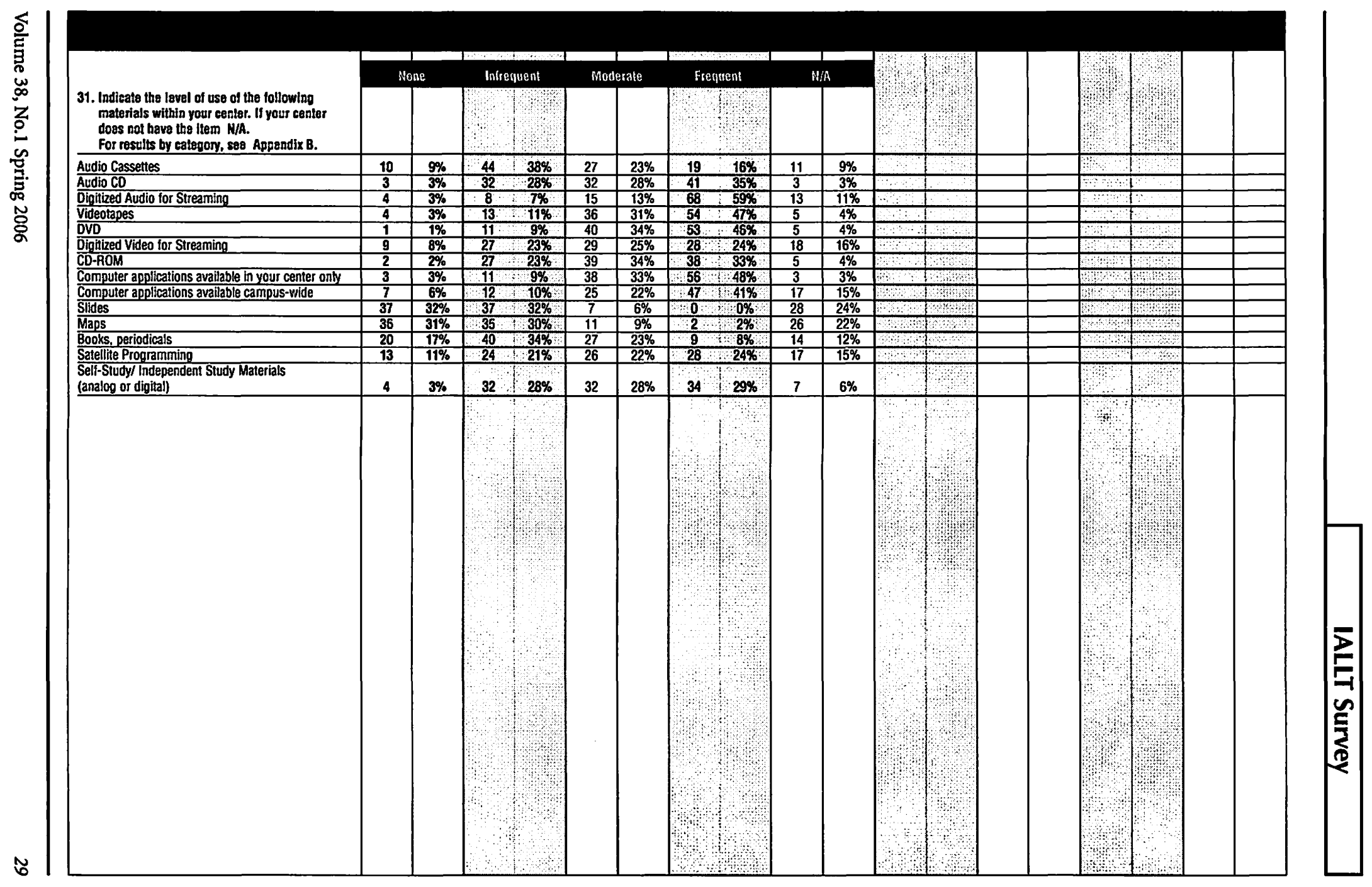




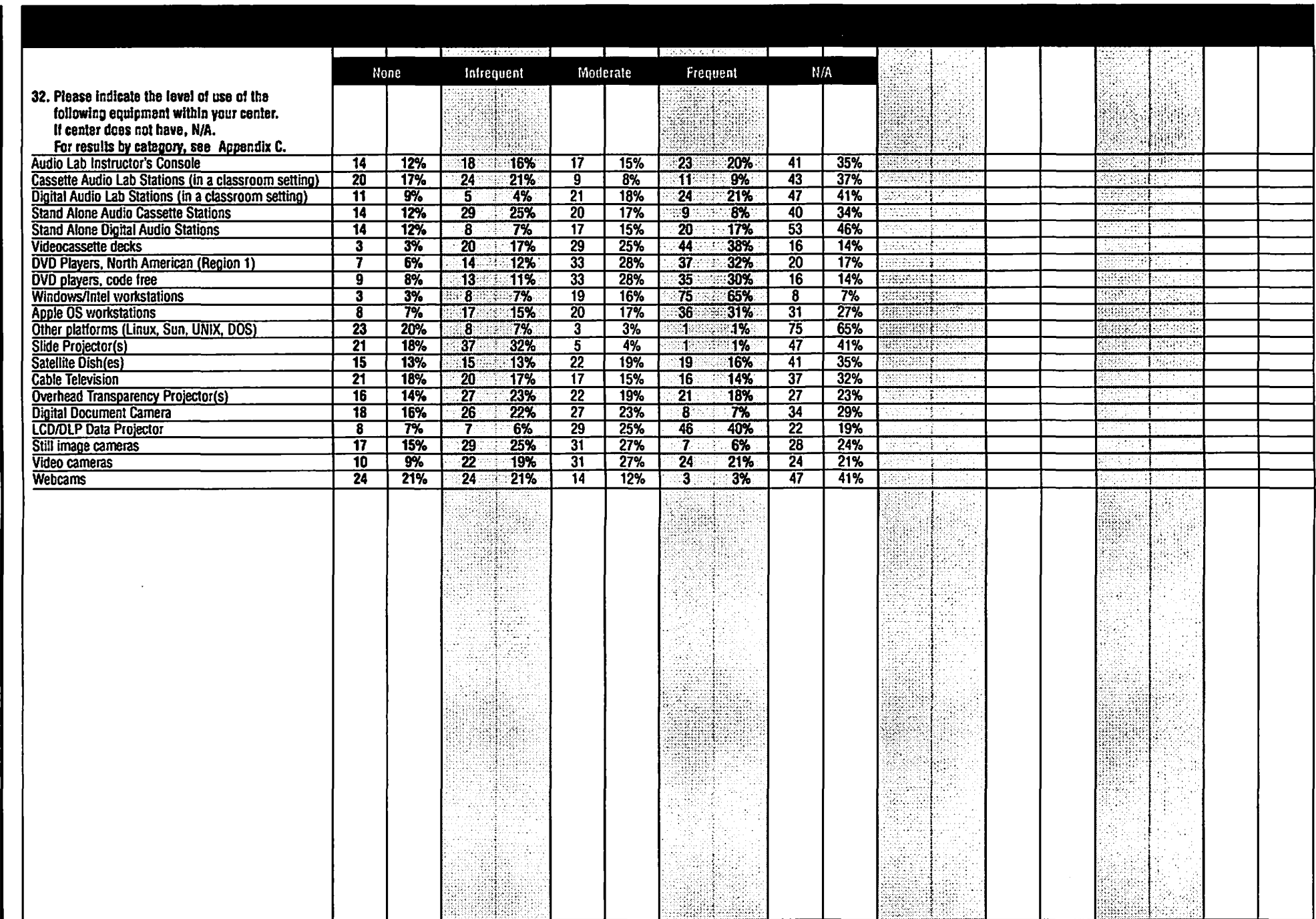




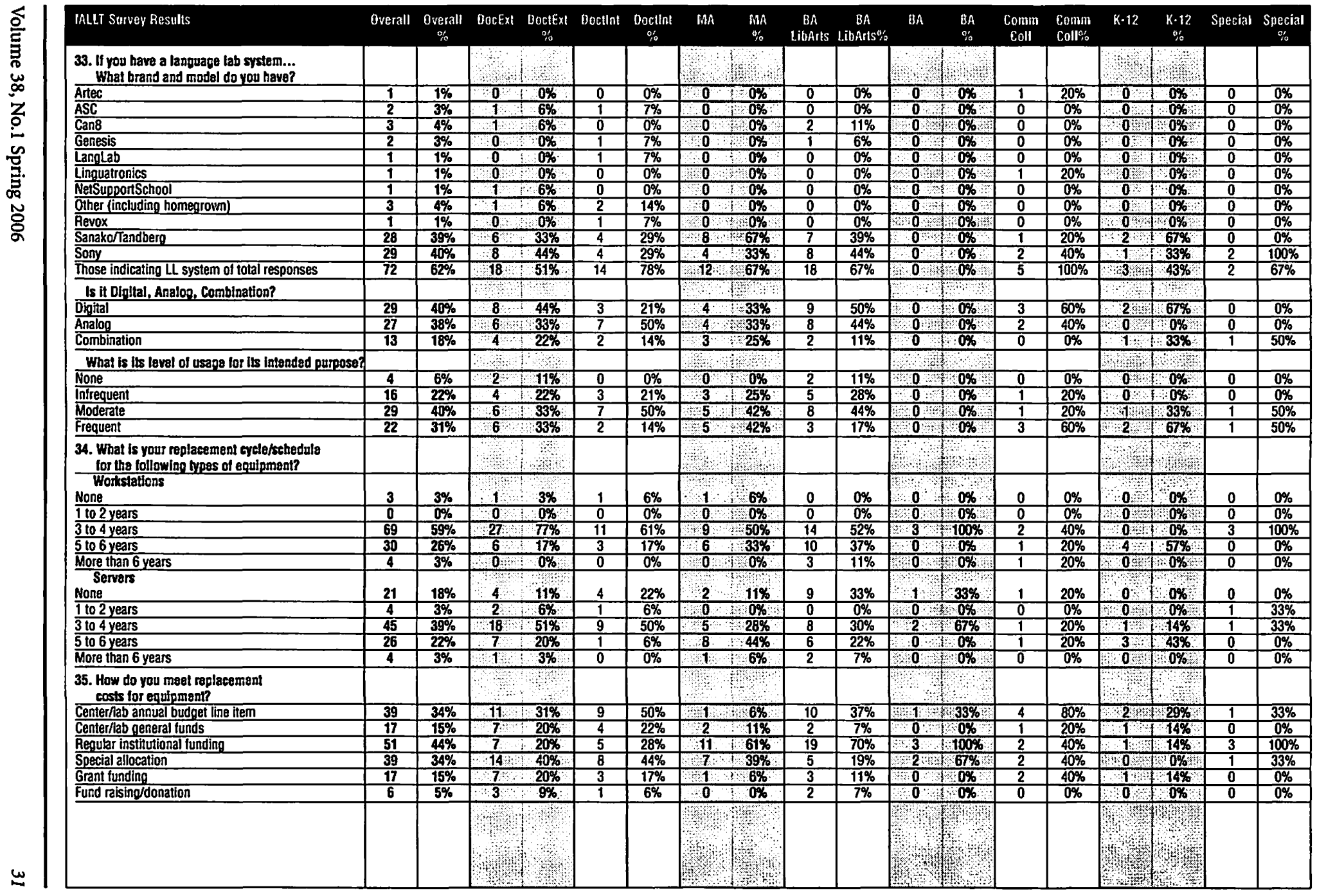




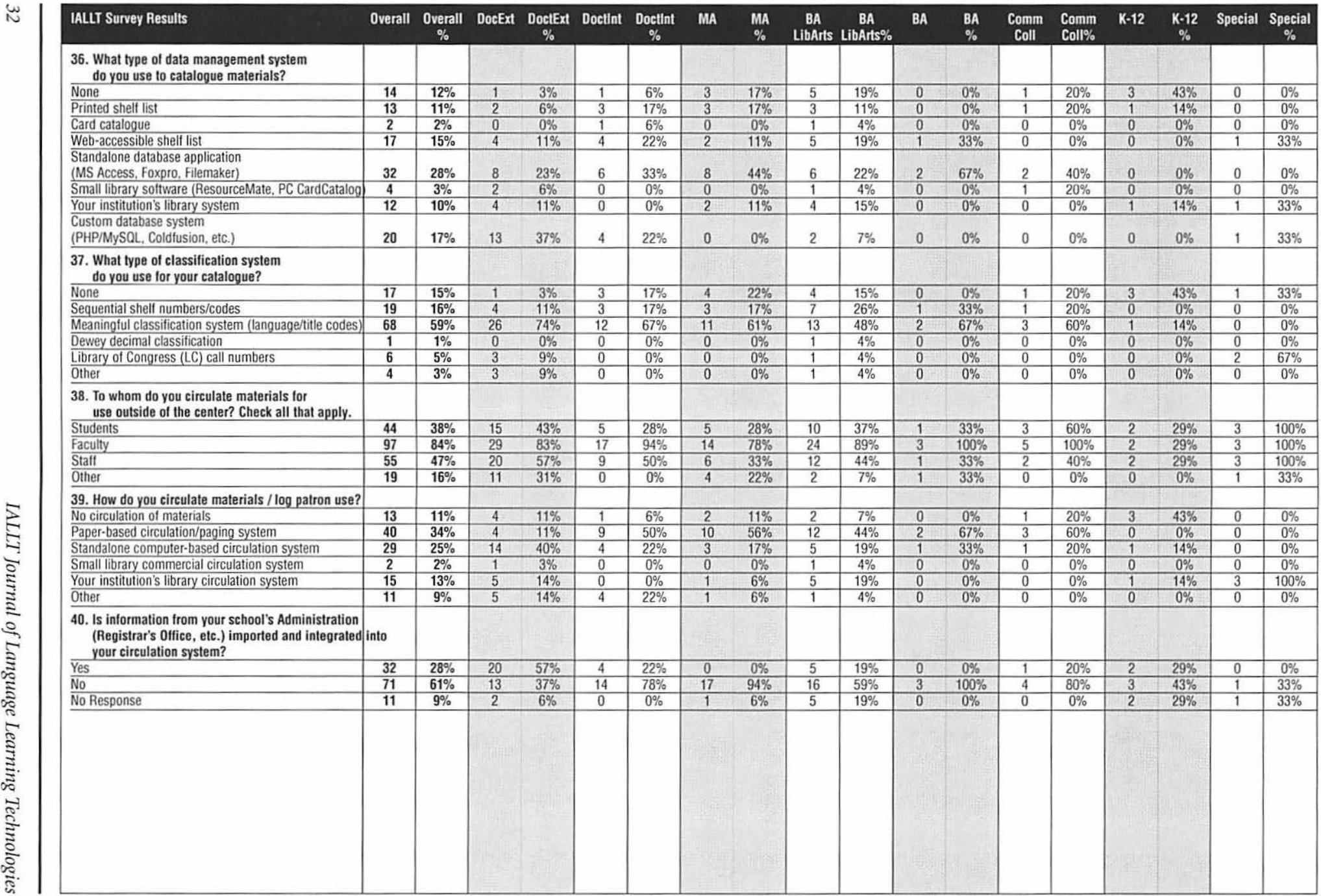



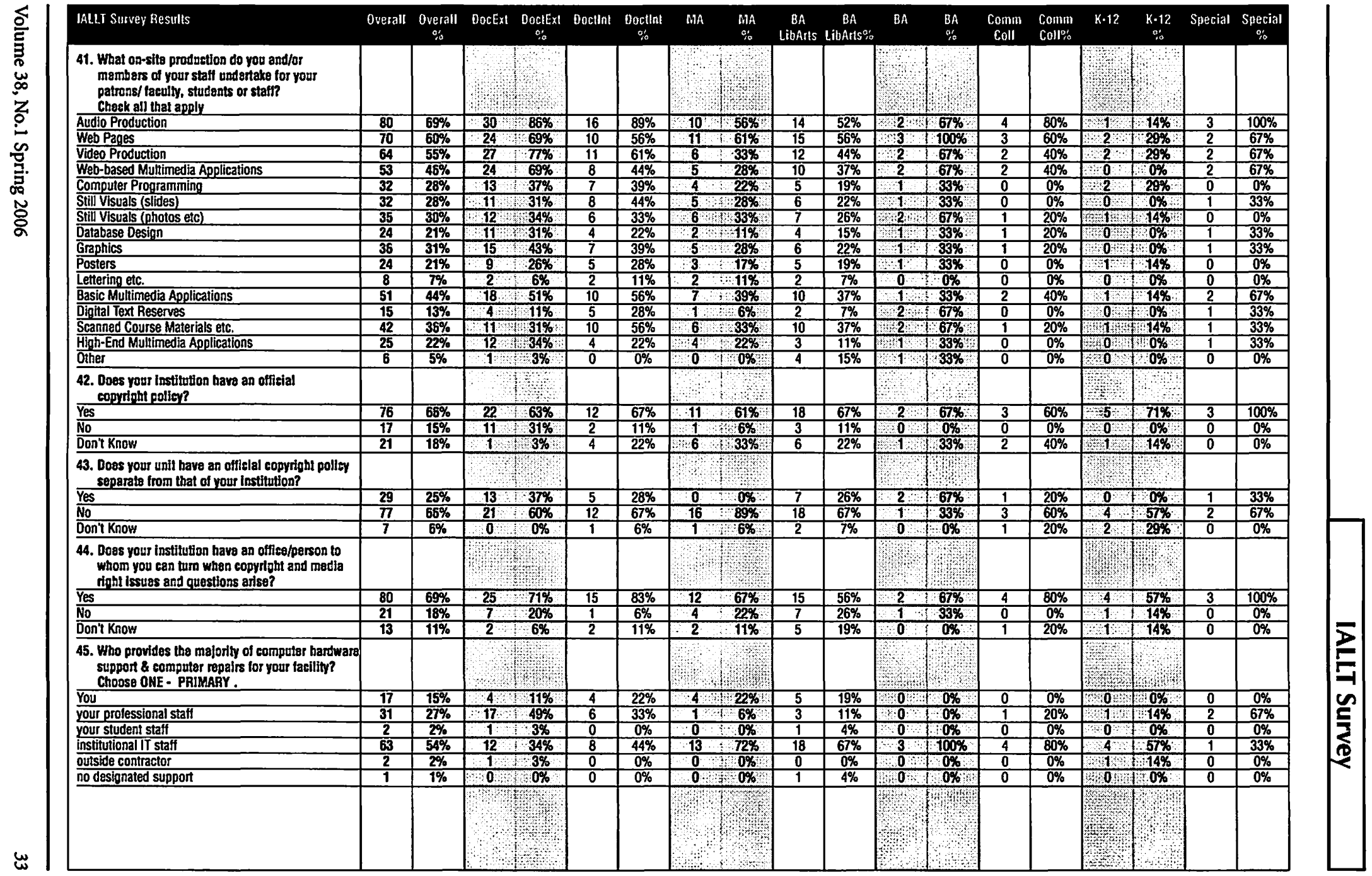
46. Who provides the majority of computer hardwar support and repairs for faculty members

in your constituency? Choose ONE - PAIMARY.

your professional staf

your student stafi

institutional IT sta

no designated support

47. To whom do you most directly report?

Chair of language department

Academic dean (e.g. of Arts and Sciences)

Administrative dean (e.g. of Student Affairs)

Chief information officer (e.g. Director of IT)

Library administrator

Chief Academic Officer (e.g. Provost)

48. Does the lab have any type of formal

standing/advisory committee?

Don't know

48a. To whom does the standing/advisory

committee report?

No response

Academic dean

Chief Information Officer

Director of Academic Center

You

48b. What does the standing/advisory committee do?

Check ALL that apply.

Supervise Operations

Make General Recommendations

Evaluate Services

Communicate with Faculty

Make Purchase Decisions

Oversee Policy

Other

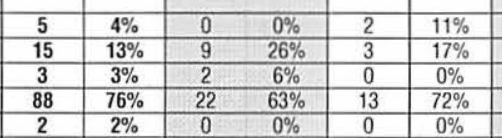

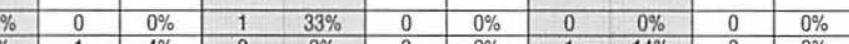

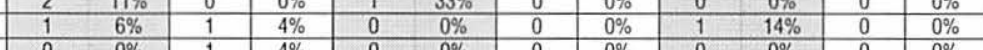
\begin{tabular}{|cc|c|c|c|}
\hline 0 & $0 \%$ & 1 & $4 \%$ \\
\hline 15 & $83 \%$ & 24 & $89 \%$ \\
\hline
\end{tabular}

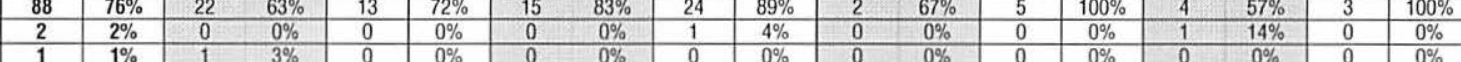

(1)

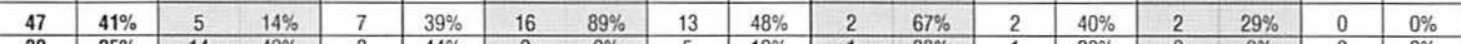

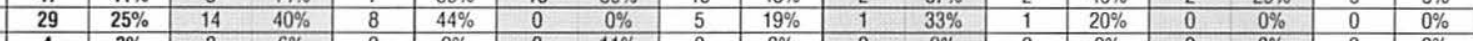

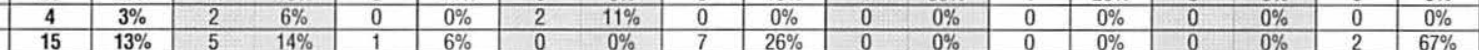
\begin{tabular}{|l|l|l|l|l|l|l|l|l|l|l|l|l|l|l|l|l}
\hline 1 & $1 \%$ & 0 & $0 \%$ & 0 & $0 \%$ & 0 & $0 \%$ & 0 & $0 \%$ & 0 & $0 \%$ & 1 & $20 \%$ & 0 & $0 \%$ & 0 \\
\hline
\end{tabular}

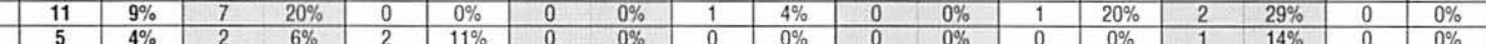

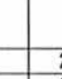

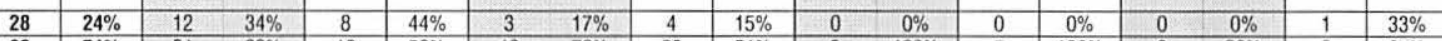

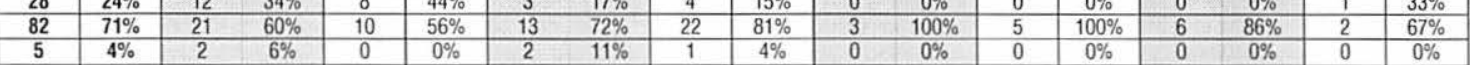

$4 \%$

(1)

\begin{tabular}{|c|c|c|c|c|c|c|c|c|c|c|c|c|c|c|c|c|c|}
\hline 75 & $65 \%$ & 24 & $69 \%$ & 11 & $61 \%$ & 15 & $83 \%$ & 22 & $81 \%$ & 3 & $100 \%$ & 0 & $0 \%$ & 0 & $0 \%$ & 0 & $0 \%$ \\
\hline 5 & $4 \%$ & 2 & $6 \%$ & 2 & $11 \%$ & 0 & $0 \%$ & 1 & $4 \%$ & 0 & $0 \%$ & 0 & $0 \%$ & 0 & $0 \%$ & 0 & $0 \%$ \\
\hline 1 & $1 \%$ & 1 & $3 \%$ & 0 & $0 \%$ & 0 & $0 \%$ & 0 & $0 \%$ & 0 & $0 \%$ & 0 & $0 \%$ & 0 & $0 \%$ & 0 & $0 \%$ \\
\hline 8 & $7 \%$ & 1 & $3 \%$ & 3 & $17 \%$ & 2 & $11 \%$ & 2 & $7 \%$ & 0 & $0 \%$ & 0 & $0 \%$ & 0 & $0 \%$ & 0 & $0 \%$ \\
\hline 2 & $2 \%$ & 1 & $3 \%$ & 0 & $0 \%$ & 0 & $0 \%$ & 0 & $0 \%$ & 0 & $0 \%$ & 0 & $0 \%$ & 0 & $0 \%$ & 1 & $33 \%$ \\
\hline 11 & $9 \%$ & 6 & $17 \%$ & 2 & $11 \%$ & 1 & $6 \%$ & 2 & $7 \%$ & 0 & $0 \%$ & 0 & $0 \%$ & 0 & $0 \%$ & 0 & $0 \%$ \\
\hline
\end{tabular}

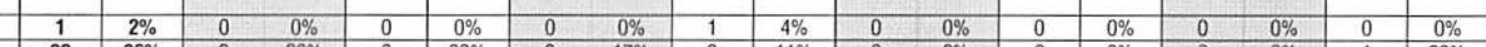

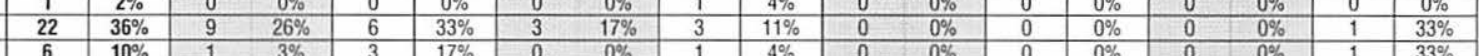

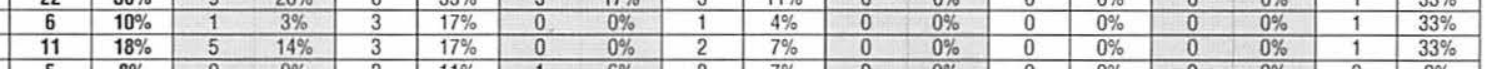

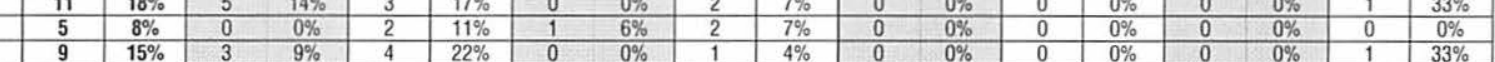
(

\begin{tabular}{l|ll|l|l|ll|l|l|}
$\mathbf{1 5} \%$ & 3 & $9 \%$ & 4 & $22 \%$ & 0 & $0 \%$ & 1 & $4 \%$ \\
\hline $\mathbf{1 1} \%$ & 2 & $6 \%$ & 1 & $6 \%$ & 0 & $0 \%$ & 3 & $11 \%$
\end{tabular}

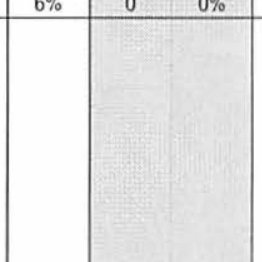




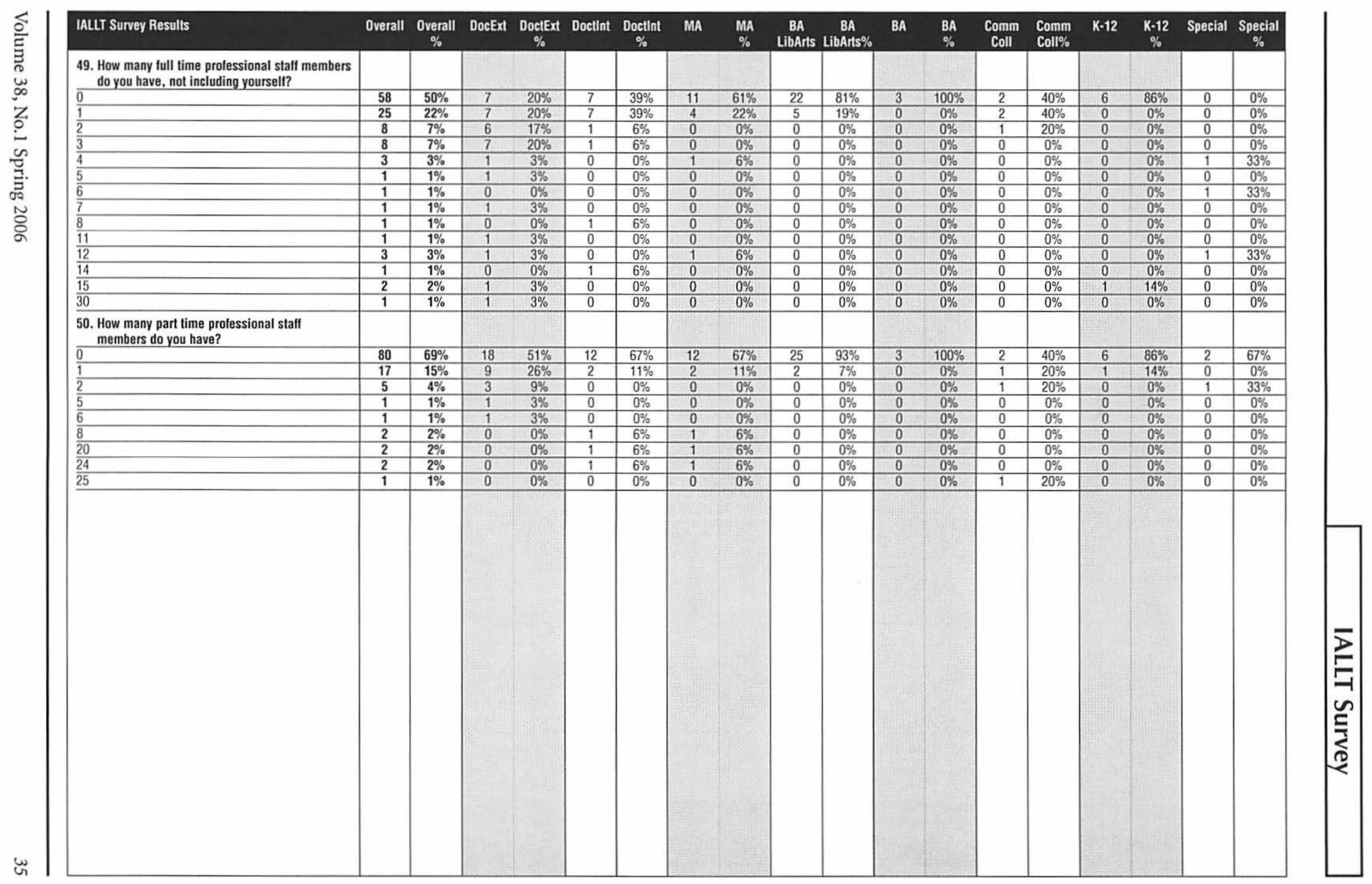




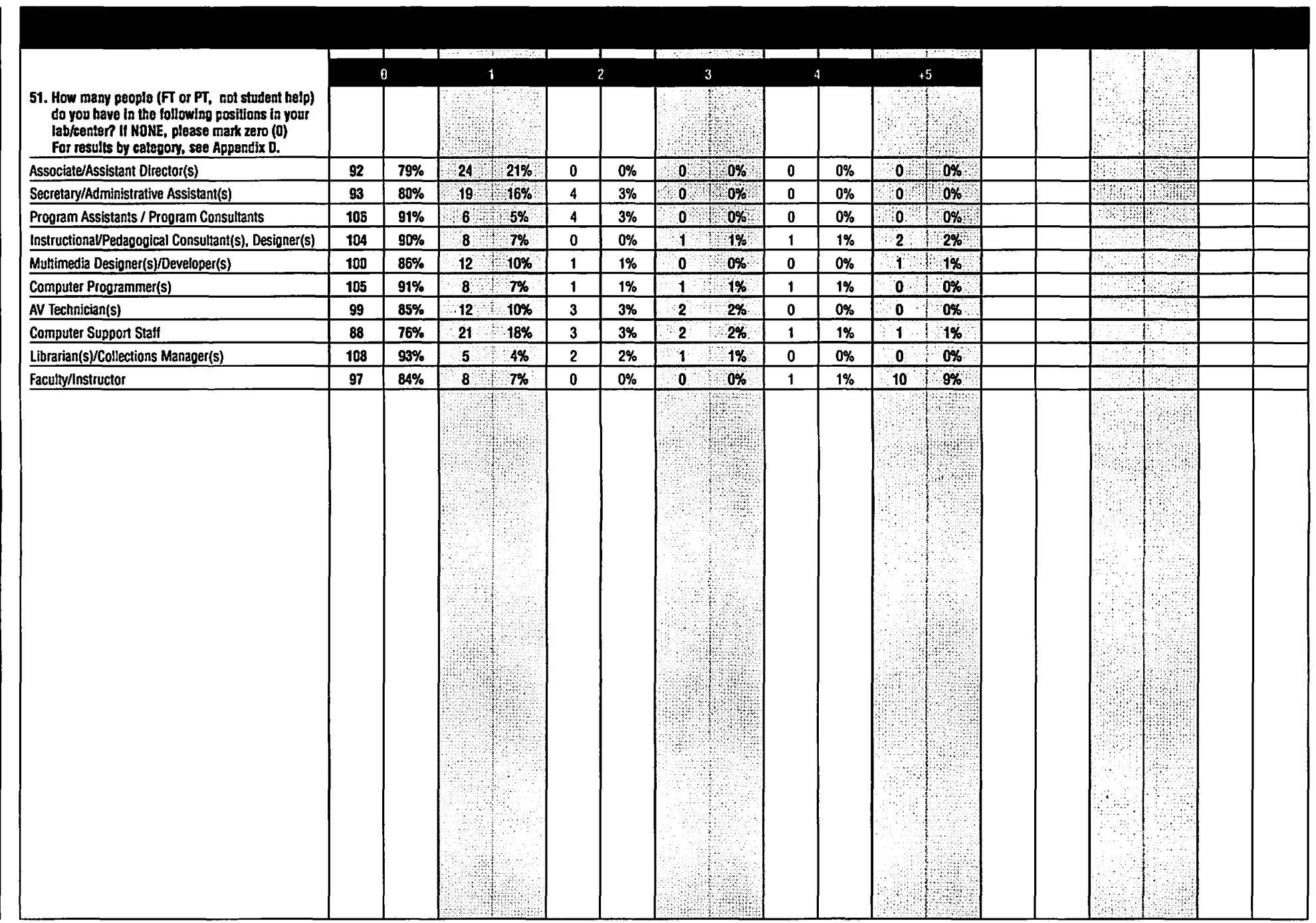




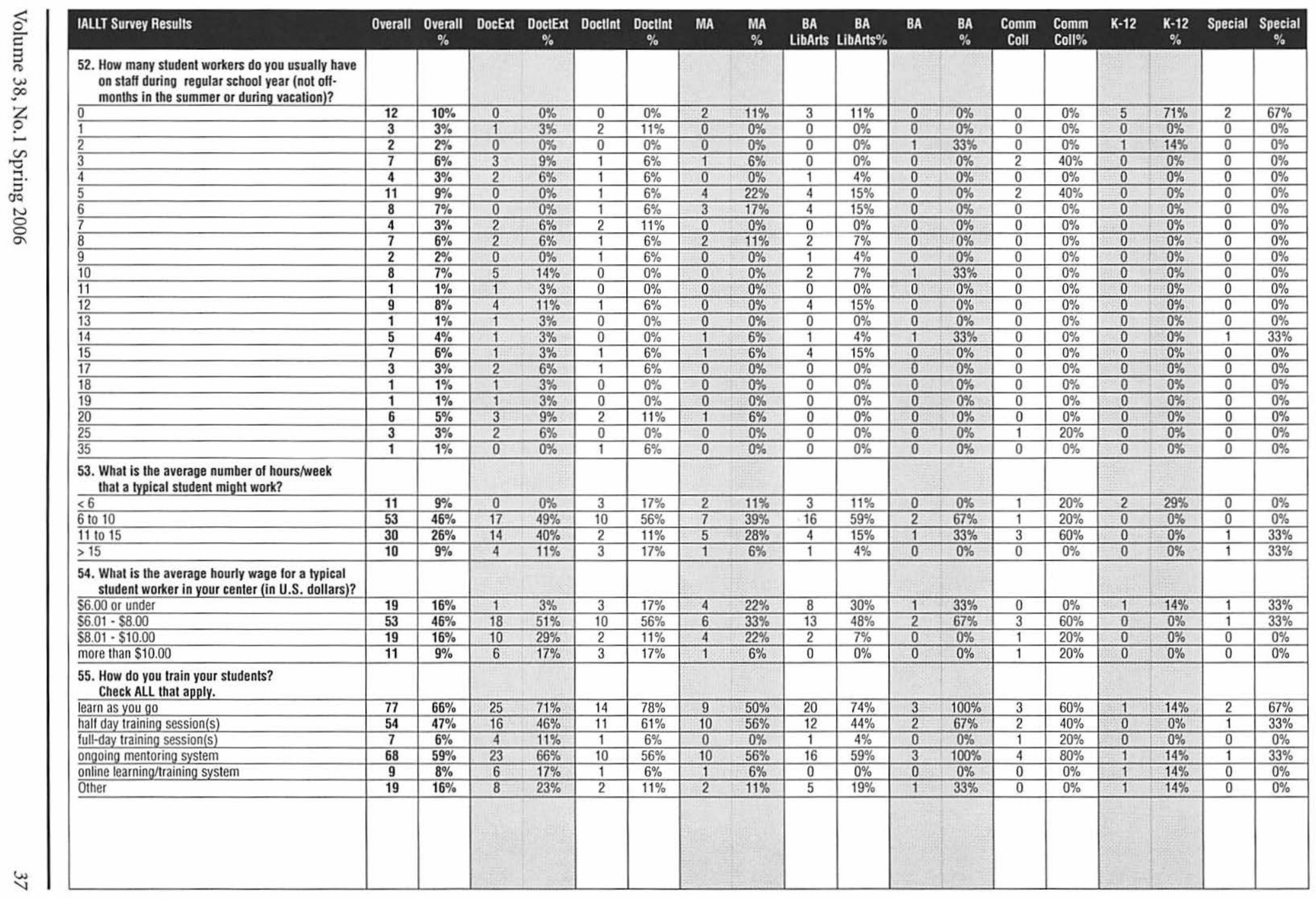




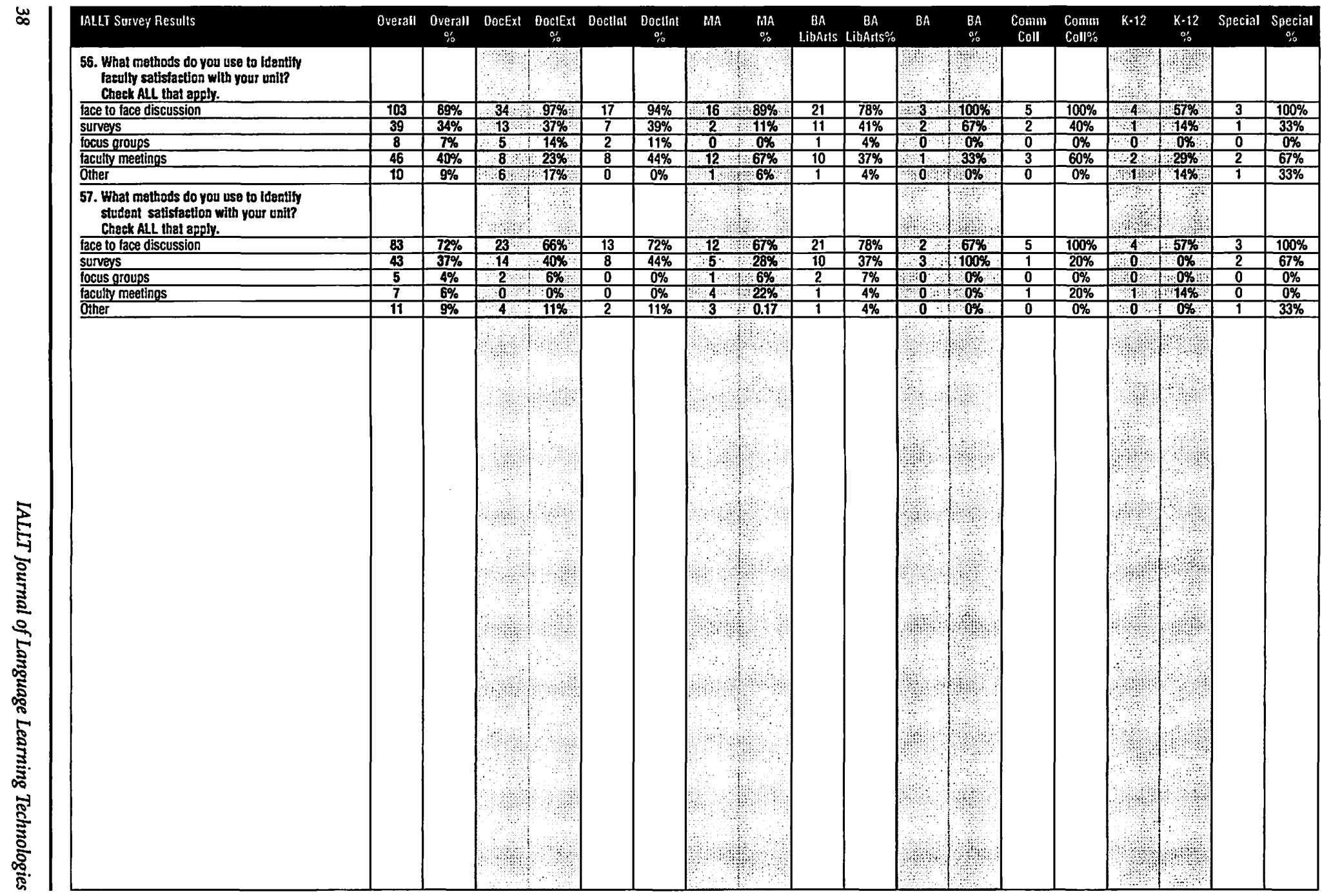

ocus groups

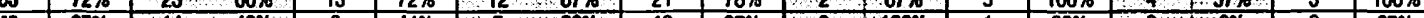

 
58. What are the most important issues your center will face during the next several years? Please use keywords only, response $=200 \mathrm{chr}$

- reallocation as a humanities lab, no longer dedicated to languages and linguistics only;

* Acquiring quality language applications and convincing faculty to use them, ${ }^{*}$ Building a quality video and audio library

1.) Analog to digital conversion of audio, slide and video materials

appropriate funding for technical/manager position and for renovatiion

Biggest issue is budget to replace computers and server, and instructional materials.

Branching out from audio delivery as publishers put this online. Move old inhouse software forward as computers stop supporting it. Offering services online-copyright and student tracking issues. Funding

Changes in technology; finding/creating adequate materials for practice, assessment; a possible swing of the pendulum AGAINST technology; staff training \& management

Changing from analog to digital labs

Changing technology

Continue integration of technology to language curriculum. Faculty training.

Continuing to keep up with technological changes to meet the needs of faculty and students in the area of language learning. Hiring a new lab director to oversee the lab. Faculty development/training.

Conversion of all materials to digital format and decision to purchase a digital lab or not. Relevance of physical space as more material is provided via web.

Converting more analog resources (equipment, holdings) to digital; restructuring staff for all-digital environment

Converting to digital equipment and media, in addition to funding these projects.

DL continues to expand, no space for additional staff; funding cuts; lab hardware/software maturation/replacement

equipment replacement

Equipment upgrade, digitization, internet-supported learning

Expanding resources beyond curricular needs to better serve the broader community. Staffing issues-increasingly difficult to sustain \& move forward with one director who also teaches.

Faculty engagement in instructional technology

funding

Funding and expansion issues. Need twice the space that we currently have. Convincing Registrarl's Office to give up classroom space and convert usage over to ILRC for expansion. 


\section{IALLT Survey}

funding, institutional support, inertia, lack of space, standards, legacy software/equipment/instructional material

funding, need for language education in space program

Funding, staff reductions, increased work load, instructor training, move to digital

FUNDING, SWITCING TO PCs (only one in 10 students on campus use Macs

Getting everyone to go from analog to digital. Finding money in budget for this change. Adhering to copyright laws in digital age.

Growth

Increase effective integraton of technology into an increasing number of foreign language AND literature AND Culture classes; research and development ; applications for linguistics;

Increase in Professional Staff

Increased enrollment has put space at a premium and will probably lead administration to close lab, given age of equipment ( $9 \mathrm{yrs}$ ) and replacement cost. Smart classrooms may assume some lab functions.

Increasing number of student-owned computers may reduce the need for center-supplied computer stations; increase of publisher-provided, online material may also reduce need for a large center.

Instituting and implementing electronic placement exams in French, Italian and Spanish.

Institutional support and funding.

insufficient human resources; lack of funding to hire faculty of any kind

Integration into IT, disolution, invasion

integration with larger campus IT structure

justifying our continued existence

Lack of adequate staffing to support our ever increasing roles in installing \& maintaining classroom tech equipment.

lack of financial support

Lack of funding and institutional support, move to another bulidling, bringing older faculty into the digital age, providing services that bring faculty into the physical center

Lack of funding for staff support and for equipment recycling. My assistant quit 2 months ago with no replacement in the near future, if at all. Too many $70 \mathrm{hr}$ work weeks, spread too thin.

Making project development more efficient, transparent for faculty. Monitor use of in-house facility as more materials move to web \& adjust resources accordingly. Make student digital video projects 
money, faculty incompetance.

Money; rapidly changing technologies, $\$$ to upgrade to these; higher expectations from users, $\$$ to satisfy expectations; national xenophobia causing atrophy of the need for language/culture skills

more faculty and students to serve, with no additional financial or administrative resources; upgrading equipment;

more off-campus delivery; disintegration of collaboration between language units; funding problems; lab renewal

need facility director with foreign language background

Need new computers; faculty training; keeping up with the latest technology

new Elem Spanish Coordinator who may not value instructional technology; relocation within 5 years \& will have to fight for space; donl't want to be absorbed by College of Liberal Arts

new university language requirements

Our center has been jumped over by textbook companies that want to move all their materials to the web and have them accessible anywhere anyone can open a web browser.

Our greatest challenge is the transition to a new facility. Also, the language requirement is \"under attackl" at the curriculum committee level.

Our high school does not have a seperate lab for foreign languages due to (surprise) spacing and budget issues. Consequently, we do not have any online or computer-related ancilliaries with our progr

Our information education center accomodates about 300 computers for classroom use and an additional about 60 computers for students use. Computer literacy,FL and other diciplines are taught.

paradigm change in language instruction, new methodologies, new concept of space, interaction of $1 / 1$, video technologies, foreign study support, assessment

Physical vs. Virtual Language Lab. What are the benefits of an actual physical location for the language lab and should be force students to go to that location top study language?

picking a digital lab system, student support with limited funding for staff, continued dual facultystaff nature of my position (constantly pulled in two directions and not covering anything well)

Possible convergence of technology and services with other campus computing centers.

Providing students with secured remote access to lab materials; securing jobs: competent technicians/consultants and full-time tenured academic position in CALL/SLAIT.

relevance expansion maintenance mission

reliable off-campus access, upgrades of digital lab system, replacement of computers and servers

Renovation of the Language Lab (going digital)

reorganization and rennovation. The building is being remodeled and we are moving to a temporary site for $1-2$ years, when we come back we will have expanded services and fewer classrooms to support. 
replacing analog lab with a multi-media computer language laboratory system, staying on top of computer systems and new technology for use in the center.

Revising mission in light of publisher-distributed materials, faculty outreach, creating production facilities, working with other administrative departments

shrinkage of the physical lab

Since more material is available online, students prefer to study in the own environs so I can see the OIT phasing this out to be just a general use lab.

Space, Money, Digital, server, streaming, relevance to university mission, continuity staffing

Staying abreast of smart classroom applications and of textbook companiesl' move online; training all partime teachers to use our dedicated course management system in full.

Student involvement comes with faculty involvement. Difficulty in motivating adjuncts to integrate lab use in their teaching. No recognition for involved faculty.

switch to digital

The change of Language Lab sessions for internationals for only one year,the following year, the international students will be in their regular classroom taking all classes as they should.

This is all new tech, instructors need to adapt to it. Also, need to develop distance learning and hybrid coursework.

Transition from a student-centered service operation to one that is faculty centered; define our position vis-a-vis other tech providers on campus; develop language research potential.

Transition from physical to virtual space.

Underpayment of students in para-professional technology jobs; - ignorance of language learning methodology and instructional technology; - Lack of administrative planning and interest in quality

updating the library

upgrade to latest technology, implementation of e-learning

Upgrading and maintaining Equipment and Systems, especially the servers.

We are currently looking at replacing our standing lab with a wireless-lab system to be used in various classrooms. All students in our school (Grades 6-12) own a wireless laptop computer for class.

We have 3 labs in 3 high schools. Replacing equipment, acquiring software \& receiving technical support will be our greatest needs in the NCLB and funding withdrawal climate in which we now work.

Web delivery of lab programs (audio and video)

Whither the language lab. 


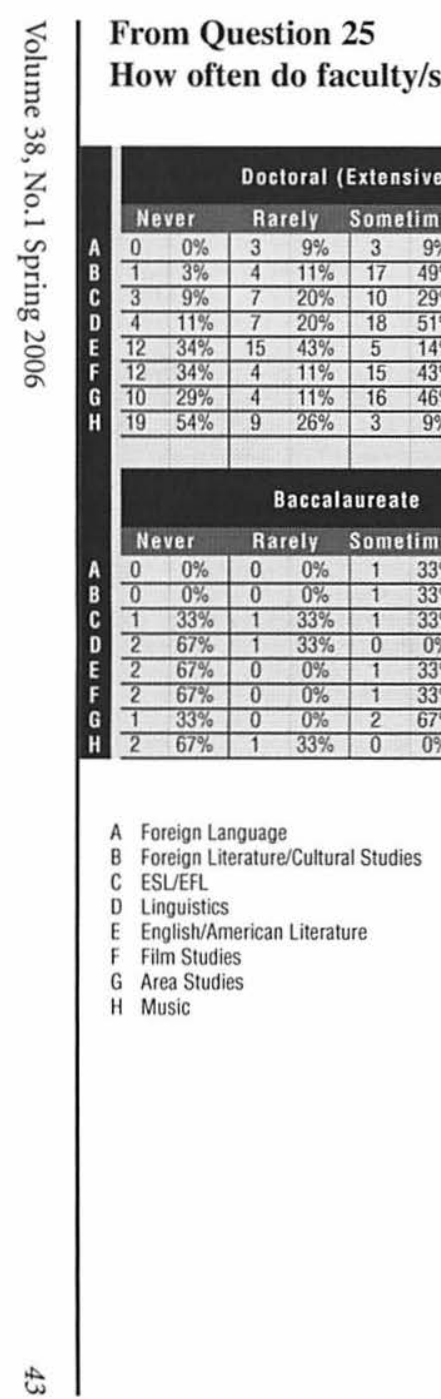




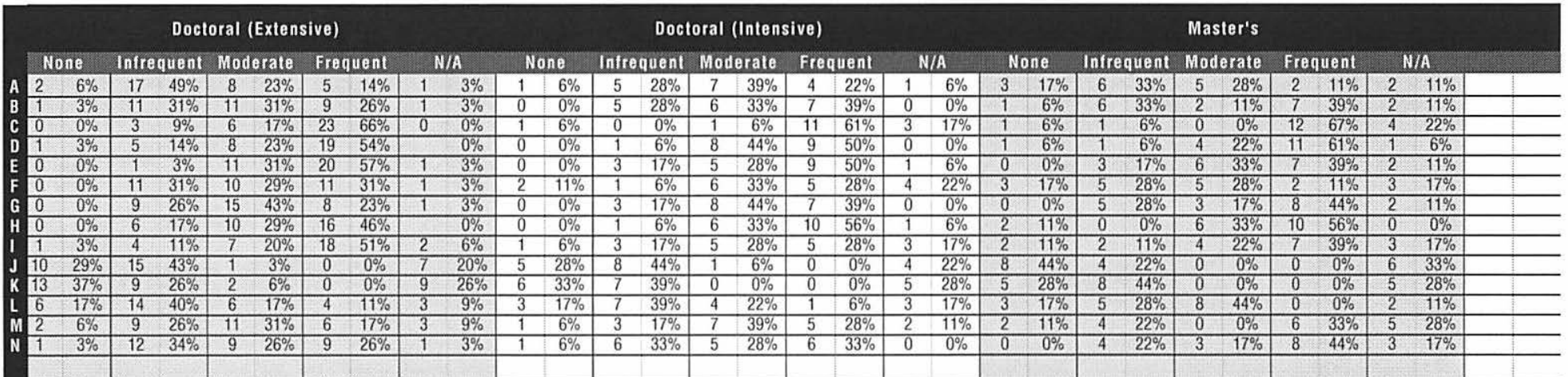

Baccalaureate (Liberal Arts)

Baccalaureate

Community College

None Infrequent Moderate Frequent N/A None Infrequent Moderate Frequent N/A None Infrequent Moderate Frequent N/A

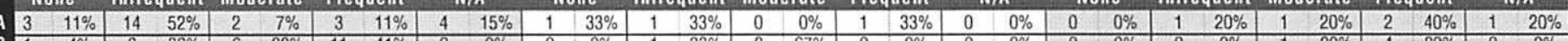

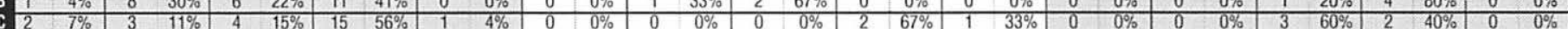

\begin{tabular}{lllllllllllllllllllllllllllllllll}
\hline & 2 & $7 \%$ & 3 & $11 \%$ & 10 & $37 \%$ & 11 & $41 \%$ & 0 & $0 \%$ & 0 & $0 \%$ & 1 & $33 \%$ & 1 & $33 \%$ & 1 & $33 \%$ & 0 & $0 \%$ & 0 & $0 \%$ & 1 & $20 \%$ & 2 & $40 \%$ & 1 & $20 \%$ & 1 & $20 \%$ \\
\hline
\end{tabular}

\begin{tabular}{|llllllllllll|lllll|lllllllllllllllllll}
\hline 1 & $4 \%$ & 3 & $11 \%$ & 9 & $33 \%$ & 12 & $44 \%$ & 0 & $0 \%$ & 0 & $0 \%$ & 0 & $0 \%$ & 2 & $67 \%$ & 1 & $33 \%$ & 0 & $0 \%$ & 0 & $0 \%$ & 0 & $0 \%$ & 3 & $60 \%$ & 2 & $40 \%$ & 0 & $0 \%$ \\
\hline 4 & $15 \%$ & 9 & $33 \%$ & 7 & $26 \%$ & 2 & $7 \%$ & 3 & $11 \%$ & 0 & $0 \%$ & 0 & $0 \%$ & 0 & $0 \%$ & 2 & $67 \%$ & 1 & $33 \%$ & 0 & $0 \%$ & 0 & $0 \%$ & 1 & $20 \%$ & 2 & $40 \%$ & 2 & $40 \%$ \\
\hline
\end{tabular}

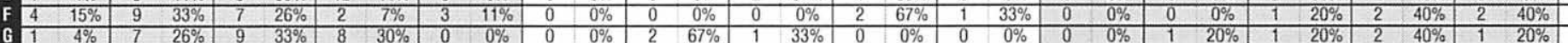

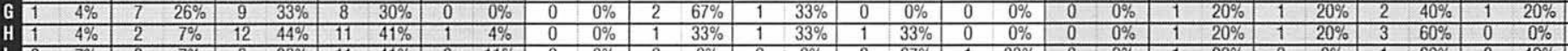

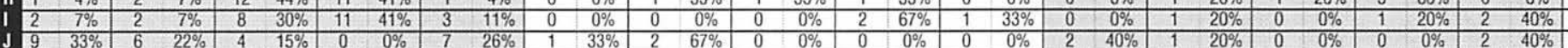

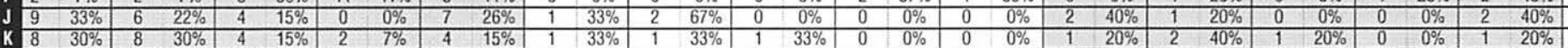

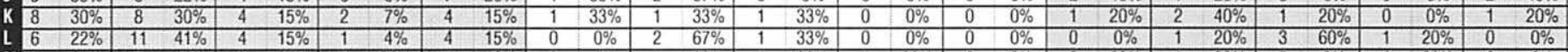

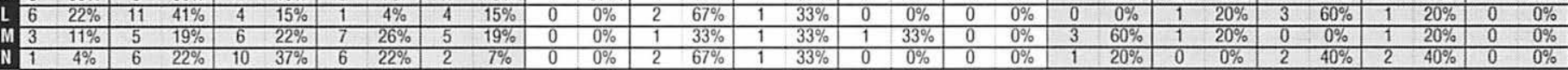

A Audio Cassettes

B Audio CD

C Digitized Audio for Streaming

$D$ Videotapes

E DVD

F Digitized Video for Streaming

G CD-ROM

Computer applications available in your center only

1 Computer applications available campus-wide

J Slides

L Books, periodicals

M Satellite ProgramminglSelf-Study/ Independent Study Materials

N (analog or digital) 


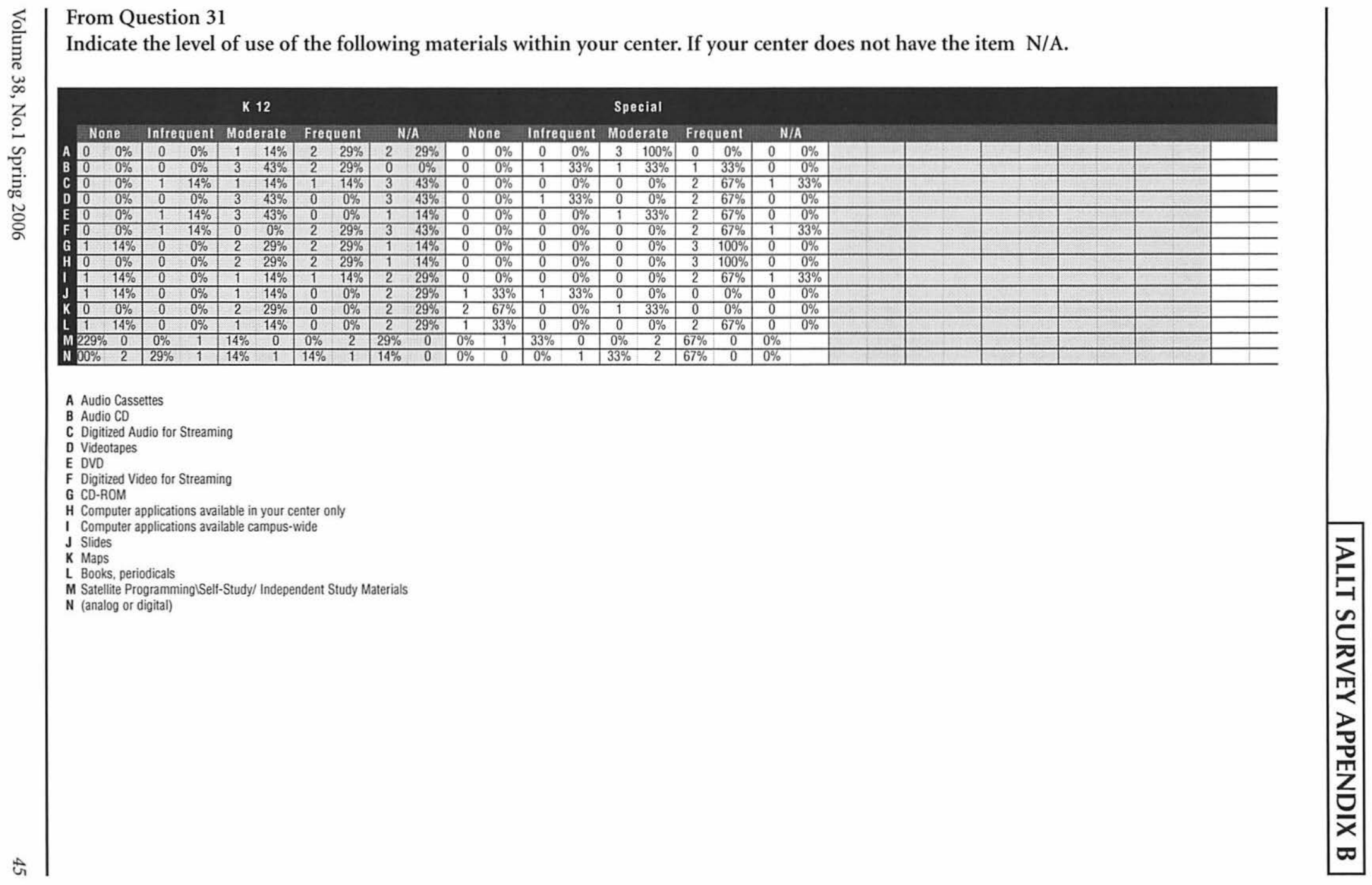


Please indicate the level of use of the following equipment within your center. If center does not have, N/A..

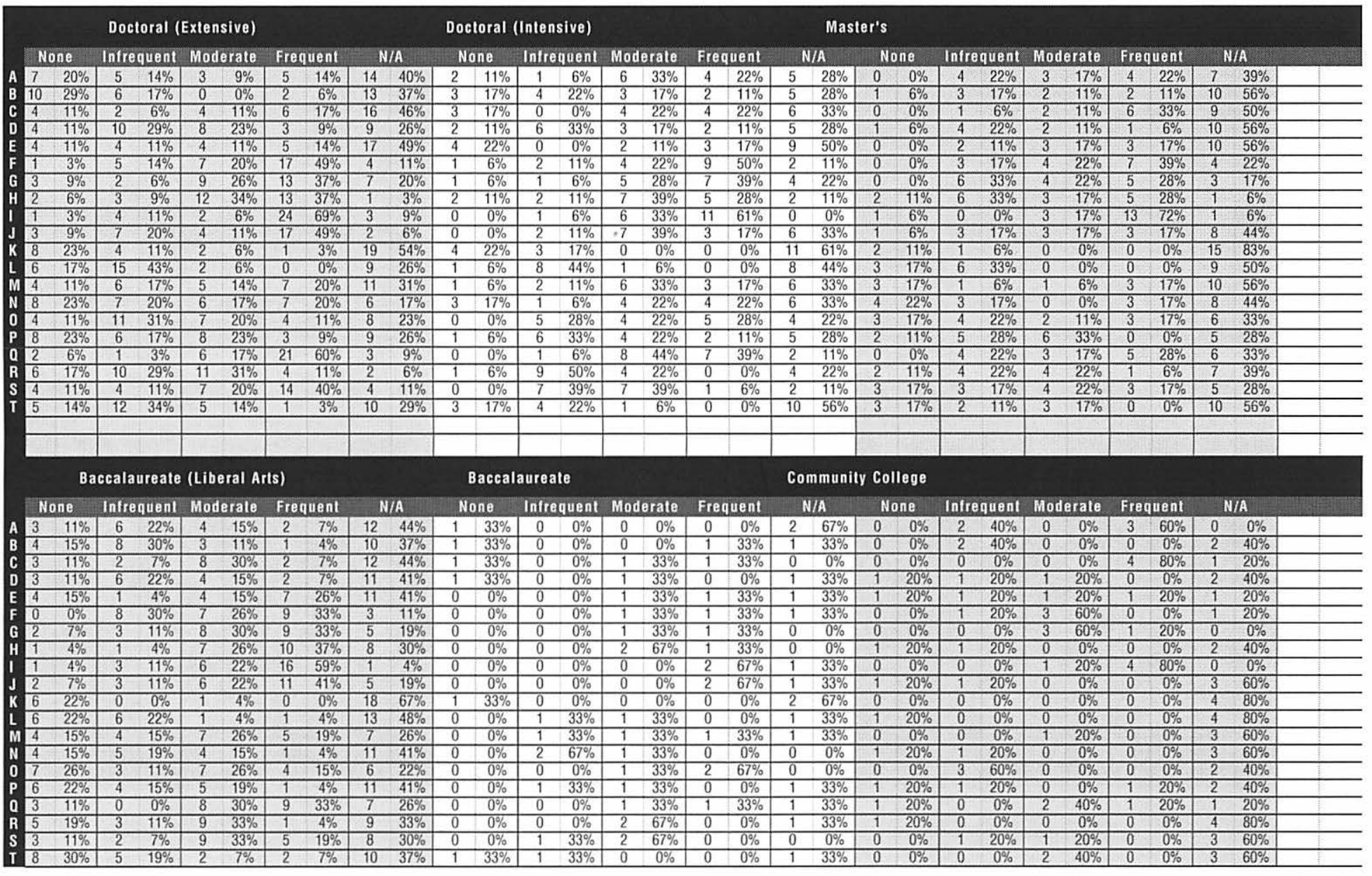


From Question 32

Please indicate the level of use of the following equipment within your center. If center does not have, N/A..

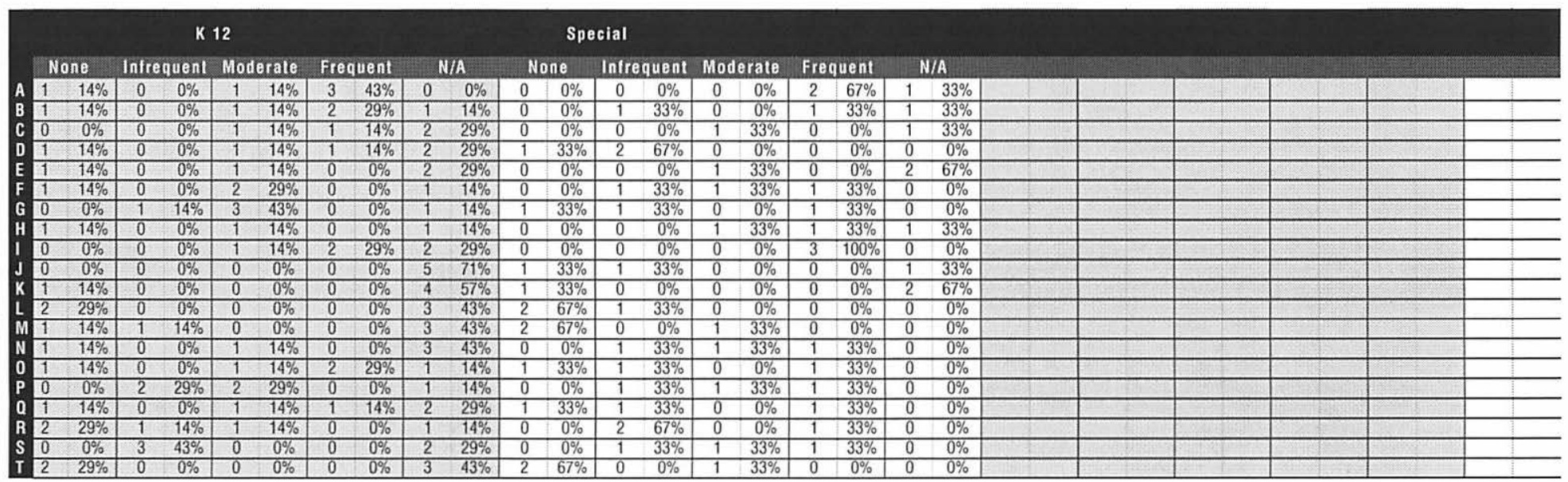

A Audio Lab Instructor's Console

B Cassette Audio Lab Stations (in a classroom setting)

C Digital Audio Lab Stations (in a classroom setting)

D Stand Alone Audio Cassette Stations

E Stand Alone Digital Audio Stations

$F$ Videocassette decks

DVD Players, North American (Region 1)

H DVD players, code free

Jindows/ntel workstations

K Other platforms (Linux, Sun, UNIX, DOS)

L Slide Projector(s)

M Satellite Dish(es)

Cable Television

P Digital Document Camera

a LCD/DLP Data Projector

R Still image cameras

$S$ Video cameras

T Webcams 

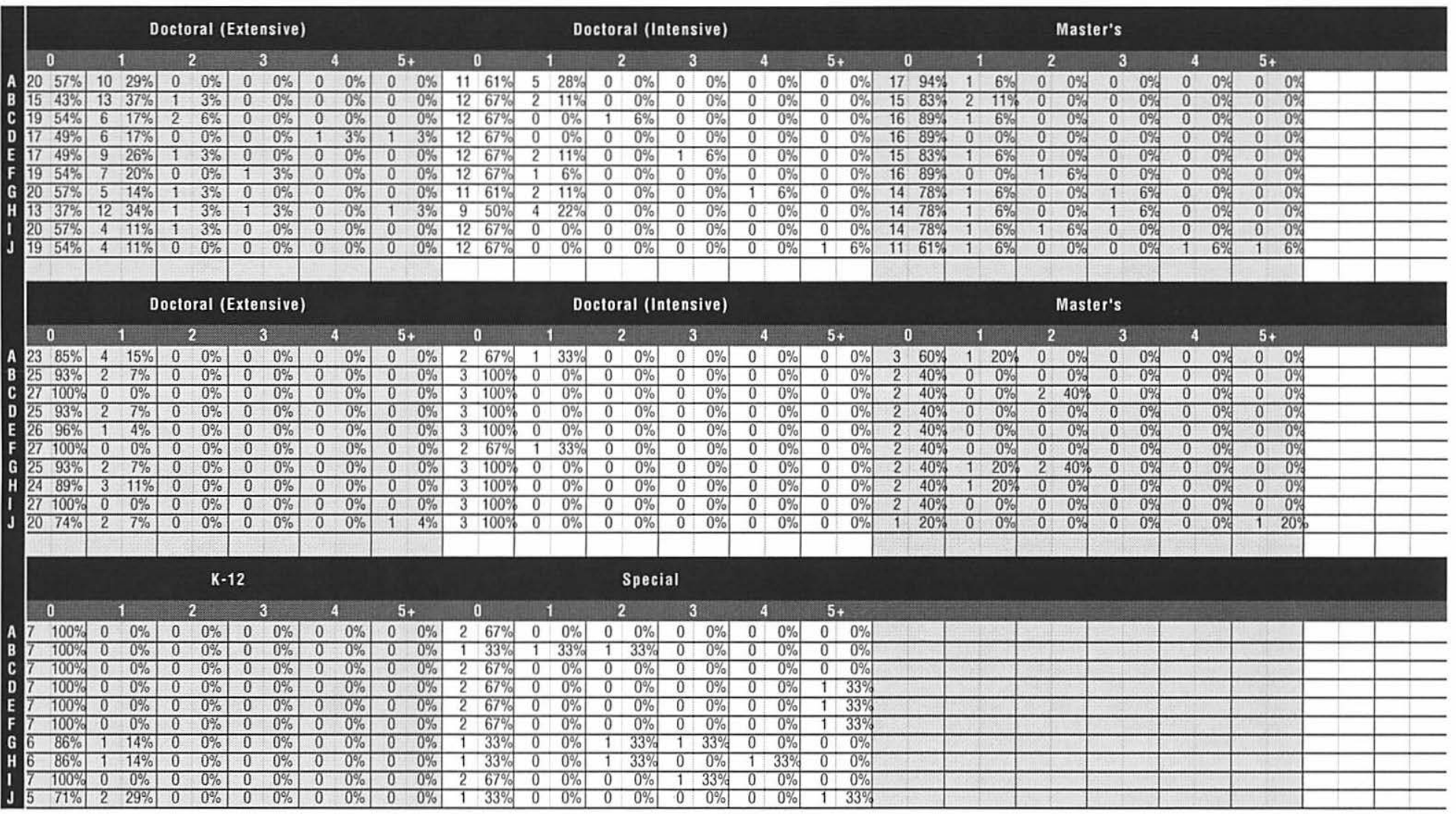

A Associate/Assistant Director(s)

B Secretary/Administrative Assistant/(s)

C Program Assistants / Program Consultants

D InstructionaVPedagogical Consultant(s), Designer(s)

E Multimedia Designer(s)/Developer(s)

$F$ Computer Programmer(s)

G AV Technician(s)

H Computer Support Staff

I Librarian(s)/Collections Manager(s)

J Faculty/Instructor 